ANL-7407

Engineering and

Equipment (TID-4500)

$A E C$ Research and

Development Report

\author{
ARGONNE NATIONAL LABORATORY \\ 9700 South Cass Avenue \\ Argonne, Illinois 60439
}

\title{
TRANSIENT COMBINED CONDUCTION AND RADIATION IN AN \\ ABSORBING NONEMITTING MEDIUM
}

by

Roger P. Heinisch and Ralph M. Singer

Reactor Engineering Division

and

Raymond Viskanta

School of Mechanical Engineering

Purdue University

January 1968

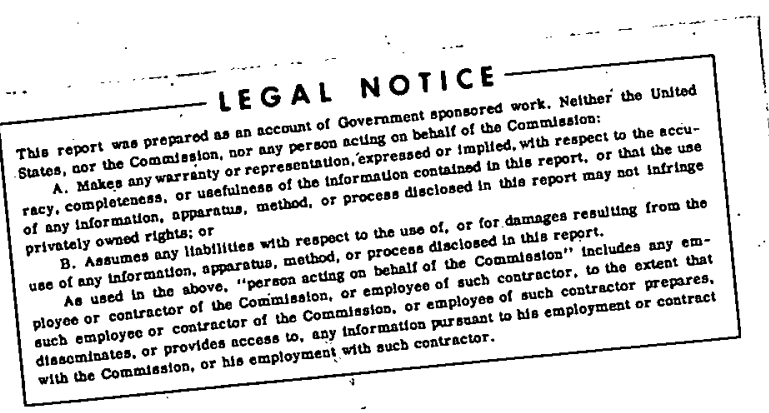




\section{DISCLAIMER}

This report was prepared as an account of work sponsored by an agency of the United States Government. Neither the United States Government nor any agency Thereof, nor any of their employees, makes any warranty, express or implied, or assumes any legal liability or responsibility for the accuracy, completeness, or usefulness of any information, apparatus, product, or process disclosed, or represents that its use would not infringe privately owned rights. Reference herein to any specific commercial product, process, or service by trade name, trademark, manufacturer, or otherwise does not necessarily constitute or imply its endorsement, recommendation, or favoring by the United States Government or any agency thereof. The views and opinions of authors expressed herein do not necessarily state or reflect those of the United States Government or any agency thereof. 


\section{DISCLAIMER}

Portions of this document may be illegible in electronic image products. Images are produced from the best available original document. 


\section{TABLE OF CONTENTS}

Page:

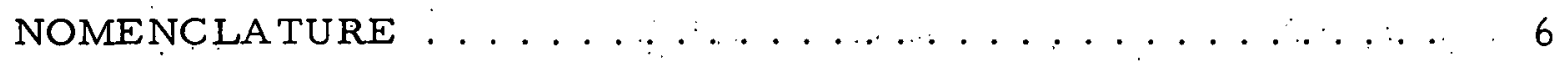

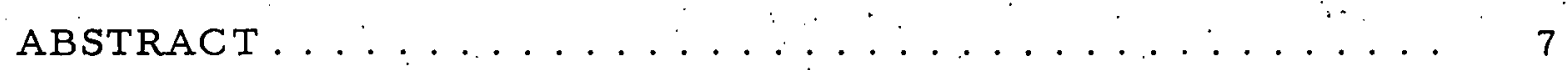

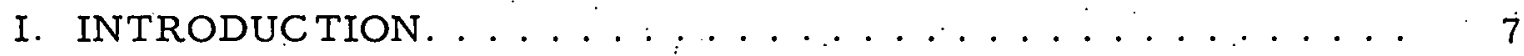

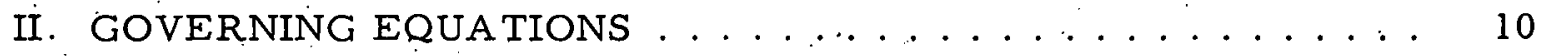

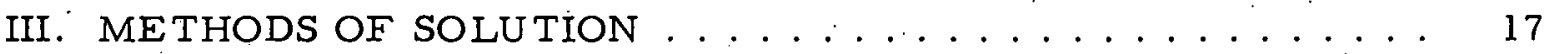

A. Superposition Method . . . . . . . . . . . 17

B. Method of Lines ...................... 20

C. Lumped-parameter Finite-difference Method ........ 22

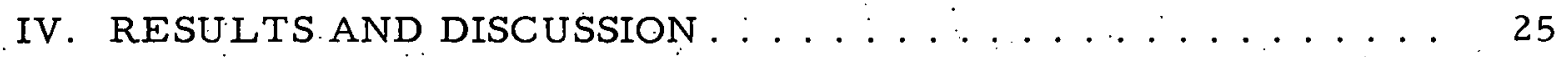

V. CONCLUSIONS AND RECOMMENDATIONS . . . . . . . . . . 29

APPENDIXES

A. Absorption Analysis. . . . . . . . . . . 32

B. Evaluation of Integrals . . . . .................. 35

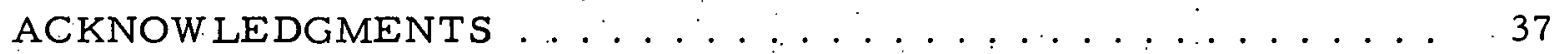

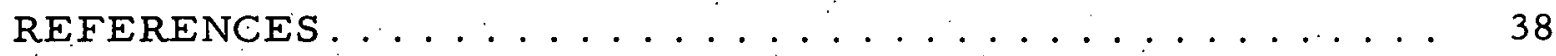




\section{LIST OF FIGURES}

No.

Title

$\underline{\text { Page }}$

1. Physical Model and Coordinate System. . . . . . . . . . 11

2. Solutions of Eq. 29 for $N=10, \tau_{0}=0.1 \ldots \ldots \ldots \ldots$

3. Solutions of Eq. 29 for $N=1 ; \tau_{0}=0.1 \ldots \ldots 25$

4. Solutions of Eq. 29 for $N=0.1, \tau_{0}=0.1 \ldots \ldots \ldots \ldots$

5. Solutions of Eq. 29 for $N=10, \tau_{0}=0.5 \ldots \ldots \ldots . \ldots . \ldots 25$

6. Solutions of Eq. 29 for $N=1, \tau_{0}=0.5 \ldots \ldots . \ldots 26$

7. Solutions of Eq. 29 for $N=0.1, \tau_{0}=0.5 \ldots \ldots 26$

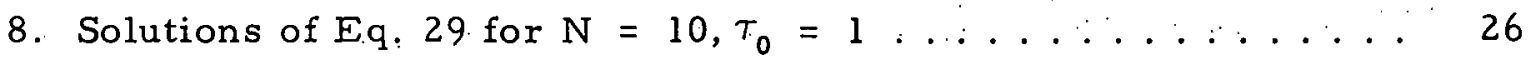

9. Solutions of Eq. 29 for $N=1, \tau_{0}=1 \ldots \ldots \ldots$

10. Solutions of Eq. 29 for $N=0.1, \tau_{0}=1 \ldots \ldots 26$

11. Solutions of Eq. 29 for $N=0.01, \tau_{0}=1 \ldots \ldots \ldots 27$

12. Solutions of Eq. 29 for $N=10, \tau_{0}=10 \ldots \ldots \ldots \ldots 27$

13. Solutions of Eq. 29 for $N=1, \tau_{0}=10 \ldots \ldots \ldots \ldots$

14. Solutions of Eq. 29 for $\mathrm{N}=0.1, \tau_{0}=10 \ldots \ldots \ldots \ldots \ldots$

15: Solutions of Eq. 29 for $N=0.01, \tau_{0}=10 \ldots 27$

16. Effect of Parameter $\mathrm{N}$ on Temperature at $\xi=0.5$ and

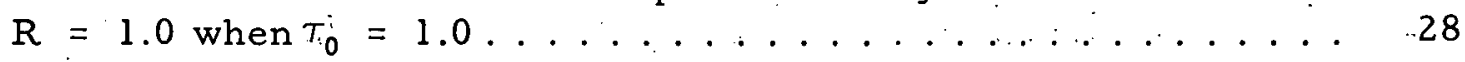

17. Absorption Cross Section of Sodium Vapor .......... 32

\section{LIST OF TABLES}

No.

$\underline{\text { Title }}$

$\underline{\text { Page }}$

I. Comparison of Numerical Values Obtained by the Three Methods at Discrete Values of the Space Variable for $\zeta=0.004$ and for Values of the Parameters $\mathrm{N}=0.1$

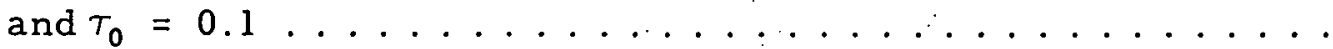

II. Comparison of Numerical Values Obtained by the Three Methods at Discrete Values of the Space Variable for $\zeta=0.04$ and for Values of the Parameters $\mathrm{N}=0.1$

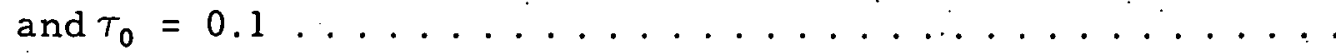


NOMENCLATURE

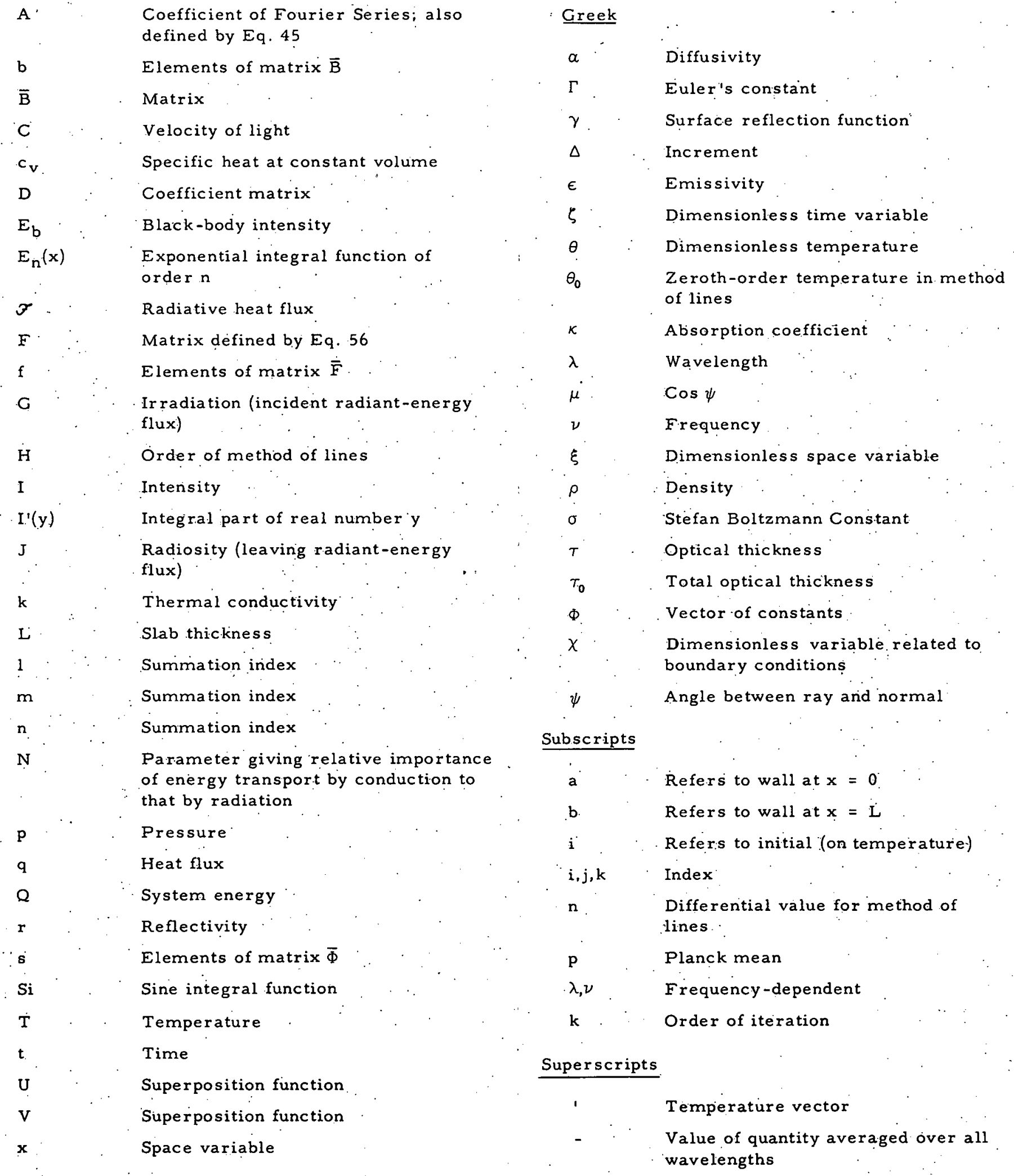




\title{
TRANSIENT COMBINED CONDUCTION \\ AND RADIATION IN AN \\ ABSORBING NONEMITTING MEDIUM
}

\author{
by \\ Roger P. Heinisch, Ralph M. Singer, \\ and Raymond Viskanta
}

\begin{abstract}
This report considers transient heat transfer by combined conduction and radiation through a plane layer of an absorbing, but nonscattering and nonemitting, stationary gray medium. The solution of the resultant linear partial differential equation is obtained in three ways: (1) an exact analytical solution in the form of an infinite series, (2) the method of lines, and (3) a lumped-parameter finite-differ ence scheme: These three techniques give essentially the same numerical results. This comparison establishes confidence in methods 2 and 3 for possible use in analyzing similar problems in which spectral and/or emission effects are accounted for. The presence of radiation as an added mechanism of energytransfer decreases the length of the transient period; an increase in optical thickness demonstrates the diffusion nature of radiation. For short times after the initiation of the transient, the solution presented is valid for the problem when the medium is a "cool" gas and the upper boundary is liquid. In particular, for a fluid such as sodium, where temperature profiles in film boiling are desired for short times following vaporization, this analysis can be used as a first approximation.
\end{abstract}

\section{INTRODUCTION}

The purpose of this investigation is to explain the mechanism of transient radiant-energy transfer in the presence of molecular conduction. The medium is a plane parallel layer that absorbs but does not emit or scatter radiation. This assumption can best be justified in the following manner: If the boundaries (or one boundary at least) áre raised suddenly to a much-higher temperature than the medium between them, then, although the medium will absorb the radiation emitted by the walls, it will not be at a high enough temperature to emit an appreciable amount of thermal radiation in comparison with the absorbed energy that originally is attributed to 
wall emission. A simple, yet effective, method of determining the validity of this approximation is to examine the fourth power of the ratio of the absolute temperatures of the fluid and the wall. When this ratio is much less than unity, the assumption is valid.

To a first approximation, the following application of the above model is proposed: During the initial transient stages of the vaporization of a layer of sodium, the vapor-liquid interface will probably not move extensively ${ }^{12}$ and thus is chosen to be stationary. One wall is held at an extremely high temperature, while the other wall (composed of an opaque liquid) is either at the saturation temperature or at some superheated temperature, which will probably be some $40-200^{\circ} \mathrm{F}$ greater than saturation. ${ }^{13}$ In general, the experimental data on the frequency-dependent absorption coefficient, $\kappa_{\nu}$, of sodium vapor indicate that unless the temperature of the vapor is exceptionally high $\left(\sim 2500^{\circ} \mathrm{F}\right)$, it will be a very weak emitter. The model proposed above is primarily applicable when the medium absorbs much more radiation than it emits.

Appendix A contains further discussion on the absorption coefficient of sodium vapor. The Planck-mean absorption coefficient is presented together with representative values of the parameter $\mathrm{N}$ (which compares the relative importance of energy transfer by molecular conduction to that by radiation). The results presented in Appendix $A$ also permit a simultaneous check on the importance of radiative transfer in sodium vapor and the possibility of an immediate interpretation of the general results obtained here.

The present analysis is an initial attempt at a gross explanation of the results of Witte ${ }^{29}$ where an extremely hot tantalum sphere $\left(\sim 3600^{\circ} \mathrm{F}\right)$ is swung on a pendulum through a pool of stationary cool liquid sodium. A first approximation for a model of the phenomenon would predict film boiling on the sphere, and if this is correct, then the omission of radiation-energy transfer could seriously affect any thermal analysis of the problem. In his basic assumptions, Witte ${ }^{29}$ considers radiative transfer to be negligible. However, it was finally postulated that forced convection was the mode of energy transfer in this experiment.

The problem that has not been discussed is the effect of propagation of a thermal wave, i.e., the velocity at which the temperature is perturbed in the medium because of the initiation of $a^{\circ}$ transient. For molecular conduction, the thermal wave is considered to be propagated via phonon conduction in solids and molecular interactions in gases. The phenomenon of phonon transfer is recognized as an acoustical-energy packet traveling through the media and thus occurs at the speed of sound in that solid. Molecular interactions within gases aresimultaneously considered to occur at the speed of sound in that gas. Thus, no matter what the substance, a thermal wave will propagate with the speed of sound when the energytransfer mecharism is due to conduction alone. 
When the primary energy-transfer mechanism is due to radiation in a gas or transparent solid, the speed of propagation of heat is much greater than the speed of sound, for a short time after the onset of heating. Thus, the pressure within the medium is unperturbed, and the energy transfer spreads through a motionless substance as a rapid thermal wave. Approximate considerations of a spherical thermal wave driven by radiation predict the velocity of propagation to be proportional to $\mathrm{T}^{4.3} \cdot{ }^{30}$. The speed of sound in a heated gas is approximately proportional to the square root of the temperature. Thus, at the onset of heating, with a large temperature difference imposed upon the system, the propagation velocity of the thermal wave is much greater than the speed of sound. After a thermal wave passes through a stationary constant-density gas, the pressure increases. In general, for a perfect gas, $p \sim \rho T$, so that eventually the pres sure and temperature profiles approximately coincide. The existence of a pressure gradient accelerates the gas, but for small times, when the thermalwave velocity is much greater than the speed of sound, the motion is imperceptible. At an intermediate time, the thermal wave reaches the second boundary and slows down because of energy loss. As a result, the velocity of the thermal wave becomes less than the velocity of sound. Finally, the processes equalize, and either a steady-state condition is met or the upper surface accelerates because of the increase in pressure.

Considerable effort has been expended in recent years on the interaction of the various modes of heat transfer. Surveys by Cess ${ }^{1}$ and Viskanta ${ }^{2}$ summarize the research performed on the interaction of radiative transfer with conduction and convection and list pertinent references.

One of the first investigations of the interaction of conduction and radiation was considered by Van der $\mathrm{Held}^{3}$ in an attempt to account for errors in thermal-conductivity measurements of fibrous materials. The solution was based on the diffusion or Rosseland approximation for the radiative flux. In a later work, Gardon ${ }^{4}$ applied a finite-difference formulation using the conservation of energy on each "node" to obtain the temperature distribution in hot glass.

Other similar analyses have been made for time-dependent gasdynamic problems; 5 but, in general, molecular conduction is neglected, while convection is not.

Lick ${ }^{6}$ investigated the problem of simultaneous conduction and radiation for a semi-infinite, gray, nonscattering, stationary medium. He solved the problem by linearizing the governing time-dependent equations and then converting the resultant integro-differential equation to a differential equation by an application of a degenerate-kernel substitution technique. Nemchinov ${ }^{7}$ analyzed a similar problem in much the same fashion, but obtained very limited results. 
Prasad ${ }^{8}$ considered the unsteady radiant heat transfer neglecting conduction in gray, nonscattering, stationary media between two parallel plates. Exact nonlinear integro-differential equations were derived and simplified for the assumptions made, and various techniques were chosen to attempt a solution.

Viskanta and Bathla ${ }^{9}$ also analyzed the unsteady energy transfer in a plane layer of radiating gas. They used exact radiative-transfer methods in the formulation and accounted for variable properties. Temperature and flux distributions were obtained as functions of time for a range of parameters and initial conditions of interest.

Cobble $e^{10}$ performed an approximate analysis of combined conduction and radiation. The absorption was assumed to follow Beer's Law; that is, it is an exponential decrease with space. This assumption neglects the angular effect and assumes that the radiation is in parallel beams. With the above assumptions, an exact solution was possible, and an analysis using a nongray absorption coefficient was completed using the tabulated values of the integrals of the Planck function. The exponential decrease of heat flux is a first approximation that appears to yield good results.

In transparent or semitransparent stationary media at high temperatures, conduction and radiation normally interact and mutually determine the temperature distribution. Inclusion of the mechanism of energy transport by radiation can cause order-of-magnitude changes in the heat flux calculated by assuming only thermal conduction. In an attempt to measure the thermal conductivity of a gas at high temperature, this phenomenon could seriously affect the results unless it is accurately accounted for. Viskanta ${ }^{11}$ gives a gray, steady-state calculation that exemplifies the above discussion. Thus we see that the inclusion of the radiative contribution to the energy transport in some cases is extremely important and must be accurately accounted for.

\section{GOVERNING EQUATIONS}

Before formulating the basic governing equations, let.us examine the physical model that has been chosen. A rigorous analysis of either the steady-state or the transient combined conduction and radiation problem should include the frequency-dependent properties. Another more basic effect neglected here is the result of re-emission by the medium. The latter restriction, if taken in the context discussed above, may be omitted effectively without drastically changing the results. The former nongray analysis, by its very nature, would introduce considerable mathematical difficulties, but would also give more physically realistic results for any specific medium. A dominant difficulty is basic to nongray analysis; that is, we must resort to the solution of specific problems. The purpose of this 
report is to obtain gross trends and to provide a physically significant standard of comparison, together with the development of techniques that may be used in the obvious nonlinear extensions of this problem. Scattering is considered to be negligible compared with absorption. This assumption is a good approximation for gases and transparent solids without particulate suspensions. The walls comprising the boundaries of the slab are assumed to be diffuse.

Finally, the assumption used in the film-boiling case of a fixed "liquid wall" in space is especially restrictive for larger values of time. However, the removal of this assumption introduces nonlinearities which by themselves are extremely difficult, if not impossible, to handle with some preset degree of accuracy. We are currently working at removing some of the aforementioned assumptions.

An exact analytical solution to the governing linear equation is obtained by a superposition technique. The exact results based on the analytical method are compared with two approximate numerical methods of solution; these comparisons indicate the accuracy of the techniques for extended use on combined-modes
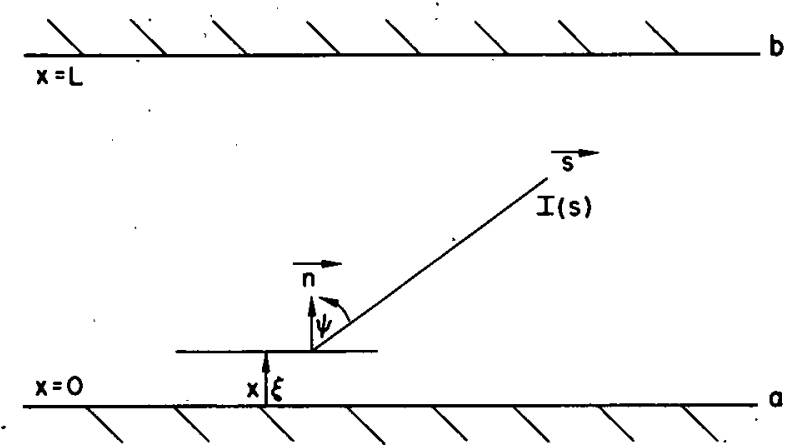

112-9187

Fig. 1. Physical Model and Coordinate System problems of the class discussed here.

Figure 1 is a schematic representation of the geometry and coordinate system of the present problem. A plane, parallel, stationary layer of an absorbing media is cooled or heated by the imposition, at either or both boundaries, of temperatures different from the initial temperature of the medium. Energy transfer by convection is assumed to be negligible compared to molecular conduction and radiative transport. Ionization and dissociation are ignored. The medium is assumed to be in local thermodynamic equilibrium and capable of absorbing energy. In the formulation of the problem, thermophysical and radiative properties are assumed constant and azimuthal symmetry is assumed. The radiant energy density. for a gas or transparent solid is much less than the internal energy density, ${ }^{1,2}$ even at extremely high temperatures, and thus is neglected. For the problem under study, in the absence of heat generation, the conservation of energy equation may be written as

$$
\rho c_{v} \frac{\partial T}{\partial t}=-\frac{\partial q}{\partial x}
$$

where $q$, the total heat flux, is defined as 


$$
q=-k \frac{\partial T}{\partial x}+\Im
$$

Equation 2 stipulates that the total heat flux is the sum of the heat transfer due to molecular conduction and that attributed to radiative-energy transfer. The radiation flux in the $x$ direction is defined as

$$
\mathcal{F}(\mathrm{x}, \mathrm{t})=\int_{0}^{\infty} \mathcal{F}_{\lambda}(\mathrm{x}, \mathrm{t}) \mathrm{d} \lambda=2 \pi \int_{0}^{\infty} \int_{-1}^{1}\left[\mathrm{I}_{\lambda}(\mathrm{x}, \mu, \mathrm{t}) \mu \mathrm{d} \mu\right] \mathrm{d} \lambda
$$

and considered positive in the positive $\mathbf{x}$ direction. The monochromatic intensity of radiation, $\mathrm{I}_{\lambda}$, is determined by the solution of the equation of transfer,

$$
\frac{1}{C} \frac{\partial I_{\lambda}}{\partial t}+\mu \frac{\partial I_{\lambda}}{\partial x}=\kappa_{\lambda}\left(\mathrm{I}_{\mathrm{b}} \lambda-\mathrm{I}_{\lambda}\right)
$$

where $\mu=\cos \psi, C$ is the velocity of light, and $\mathrm{I}_{\mathrm{b} \lambda}$ is the source function. Even if the intensity varies rapidly with time, the factor $1 / \mathrm{C}$ in the first term on the left-hand side of Eq. 4 renders the entire term negligible relative to the other terms. Thus the radiation field is considered quasistationary, but, since $I_{b} \lambda$ is a function of temperature, $I_{\lambda}$ also depends on time.

For a step change in wall temperature, the boundary and initial conditions for Eq. 1 are written as

$$
\left.\begin{array}{lll}
t=0, & 0 \leq x \leq L, & T=T_{i} ; \\
x=0, & t>0, & T=T_{a} ; \\
x=L, & t \rightarrow 00, & T=T_{b} .
\end{array}\right\}
$$

Once boundary conditions are specified for Eq. 4, a solution may then be obtained at least formally if not specifically. Considering the wall properties to be a function of $\mu$, we obtain for the boundary conditions for the intensity after integration over all frequencies.

$$
\mathrm{I}^{+}(0, \mu)=2 \mathbf{r}_{\mathrm{a}} \int_{0}^{1} \mathrm{I}^{-}\left(0,-\mu^{\prime}\right) \gamma_{\mathrm{a}}\left(\mu, \mu^{\prime}\right) \mu^{\prime} \mathrm{d} \mu^{\prime}+\epsilon_{\mathrm{a}}(\mu) \mathrm{I}_{\mathrm{ba}},
$$

and

$$
\mathrm{I}^{-}\left(\tau_{0}, \mu\right)=2 \mathrm{r}_{\mathrm{b}} \int_{0}^{\mathrm{i}} \mathrm{I}^{+}\left(\dot{\tau}_{0},+\mu^{\prime}\right) \gamma_{\mathrm{b}}\left(\mu, \mu^{\prime}\right) \mu^{\prime} \mathrm{d} \mu^{\prime}+\epsilon_{\mathrm{b}}(\mu) \mathrm{I}_{\mathrm{bb}},
$$

where $\epsilon$, the emissivity, is generally a function of $\mu$, and $r$ is the wall reflectivity. 


$$
I_{b}=\int_{J}^{\infty} I_{b \lambda} d \lambda
$$

is the total black-body emissive power, and $\gamma\left(\mu, \mu^{\prime}\right)$ is the surface reflection function characterizing the angular distribution of radiant energy. Physically we can interpret Eqs. 6 and 7 as follows: The intensity leaving a wall equals the reflected portion of the incident intensity integrated over all angles, plus that emitted by the wall. The real problem is to obtain an accurate representation of the surface-reflection function since it is a function of many parameters, such as material, surface finish, and roughness.

With the assumption of diffuse walls, we obtain

$$
\mathrm{I}^{+}(0, \mu)=\frac{\mathrm{J}_{\mathrm{a}}}{\pi}, \quad \mu \geq 0,
$$

and

$$
\mathrm{I}^{-}\left(\tau_{0}, \mu\right)=\frac{\mathrm{J}_{\mathrm{b}}}{\pi}, \quad \mu \leq 0,
$$

where $J$ is the radiosity and is defined as the sum of the emitted and reflected energy.

Formally, we can obtain the solution of Eq. 4 that results in the following expressions for the intensity for the gray case in the + and directions:

$$
\mathrm{I}^{+}(\tau, \mu)=\mathrm{I}^{+}(0, \mu) \exp \left(-\frac{\tau}{\mu}\right)+\int_{0}^{\tau} \frac{\mathrm{I}_{\mathrm{b}}(\mathrm{t})}{\mu} \exp \left(-\frac{\tau-\mathrm{t}}{\mu}\right) \mathrm{dt}
$$

and

$$
I^{-}(\tau, \mu)=I^{-}\left(\tau_{0}, \mu\right) \exp \frac{\tau_{0}-\tau}{\mu}-\int_{\tau}^{\tau_{0}} \frac{I_{b}(t)}{\mu} \exp \left(-\frac{t-\tau}{\mu}\right) d t
$$

The optical thickness is defined as

$$
\tau=\int_{0}^{\mathbf{x}} \kappa \mathrm{d} \mathbf{x},
$$

and the total optical thickness as

$$
\tau_{0}=\int_{0}^{L} \kappa d x
$$


The local radiant flux in the medium for the gray approximation is given by

$$
\begin{aligned}
& \mathcal{F}=2\left\{\int_{0}^{1}\left[\mathrm{~J}_{\mathrm{a}} \exp \left(-\frac{\tau}{\mu}\right)+\int_{0}^{T} \frac{\mathrm{I}_{\mathrm{b}}(\mathrm{t})}{\mu} \exp \left(-\frac{\tau-\mathrm{t}}{\mu}\right) \mathrm{dt}\right] \mu \mathrm{d} \mu\right. \\
& \left.\quad-\int_{0}^{1}\left[\mathrm{~J}_{\mathrm{b}} \exp \left(-\frac{\tau_{0}-\mathrm{t}}{\mu}\right)-\int_{\tau}^{\tau_{0}} \frac{\mathrm{Ib}(\mathrm{t})}{\mu} \exp \left(-\frac{\mathrm{t}-\tau}{\mu}\right) \mu \mathrm{d} \mu\right]\right\}
\end{aligned}
$$

Before performing the indicated integrations in Eq. 14, let us look at the physical interpretation of the terms involved. The first and third terms on the right-hand side of Eq. 14 represent the contributions to the flux by the energy emitted by the wall at $x=0$ and $x=L$, respectively. The second and fourth terms arise from the energy emitted within the medium. If the medium is at a much lower temperature than the walls, then the energy emitted by the medium is much less than the energy emitted by the walls and may be neglected. With this approximation, the second and fourth terms in Eq. 14 may be neglected in comparison with the first and third terms. Finally, differentiating the radiative flux with respect to the optical thickness, and carrying out the integrations, we obtain

$$
-\frac{\mathrm{d} \mathcal{F}}{\mathrm{dx}}=2 \kappa\left[\mathrm{J}_{\mathrm{a}} \mathrm{E}_{2}(\tau)+\mathrm{J}_{\mathrm{b}} \mathrm{E}_{2}\left(\tau_{0}-\tau\right)\right]
$$

where $E_{n}(t)$ are the exponential integral functions defined as

$$
E_{n}(t)=\int_{0}^{1} \mu^{n-2} e^{-t / \mu} d \mu
$$

These functions are tabulated by Kourganoff. ${ }^{15^{\circ}}$ Series expansions for the general and limiting cases are presented by Gradshteyn and Ryzhyk. ${ }^{16}$

Thus, the conservation-of-energy Eq. 1 , can now be expressed as

$$
\rho c_{v} \frac{\partial T}{\partial t}=\frac{\partial}{\partial x}\left\{k \frac{\partial T}{\partial x}\right\}+2 \kappa\left\{J_{a} E_{2}(\tau)+J_{b} E_{2}\left(\tau_{0}-\tau\right)\right\}
$$

by use of Eqs. 2 and 15. In Eq. 17, the radiation appears as a heat-source term, which physically is exactly how it interacts ${ }^{1}$ with the media due to absorption. By introduction of the dimensionless variables

$$
\zeta=\kappa^{2} \alpha t, \quad \theta=\frac{\mathrm{T}-\mathrm{T}_{\mathrm{a}}}{\mathrm{T}_{\mathrm{b}}-\mathrm{T}_{\mathrm{a}}}, \quad \xi=\frac{\mathrm{x}}{\mathrm{L}},
$$

the energy equation, in dimensionless form, for the constant-property case becomes 


$$
\frac{\partial \theta}{\partial \zeta}=\frac{1}{\tau_{0}^{2}} \frac{\partial^{2} \theta}{\partial \xi^{2}}+\frac{2}{\kappa k T_{i}}\left\{J_{a} E_{2}(\tau)+J_{b} E_{2}\left(\tau_{0}-\tau\right)\right\}
$$

The net radiant-heat flux can be obtained by specializing Eq. 14 at the surface $\tau=\xi=0$, giving

$$
\mathcal{F}=\mathrm{J}_{\mathrm{a}}-2 \mathrm{~J}_{\mathrm{b}} \mathrm{E}_{3}\left(\tau_{0}\right)
$$

with $E_{3}(0)=1 / 2$.

The first term in Eq. 17 represents radiant energy leaving surface a, and the second term is that radiant energy incident from surface b. Now,

$$
\mathrm{J}_{\mathrm{a}}=\epsilon_{\mathrm{a}} \mathrm{E}_{\mathrm{ba}}+\left(1-\epsilon_{\mathrm{a}}\right) \mathrm{G}_{\mathrm{a}}
$$

where $G_{a}=$ irradiation at a. But from Eq. 17 and the discussion thereafter,

$$
G_{a}=2 j_{b}\left(\tau_{0}\right) E_{3}\left(\tau_{0}\right)
$$

Thus,

$$
\mathrm{J}_{\mathrm{a}}=\frac{\epsilon_{\mathrm{a}} E_{\mathrm{ba}}+2\left(1-\epsilon_{\mathrm{a}}\right) \epsilon_{\mathrm{b}} E_{3}\left(\tau_{0}\right) E_{\mathrm{bb}}}{1-4\left(1-\epsilon_{\mathrm{a}}\right)\left(1-\epsilon_{\mathrm{b}}\right) \mathrm{E}_{3}^{2}\left(\tau_{0}\right)}
$$

and

$$
J_{b}=\frac{\epsilon_{b} E_{b b}+2\left(1-\epsilon_{b}\right) \epsilon_{a} E_{3}\left(\tau_{0}\right) E_{b a}}{1-4\left(1-\epsilon_{a}\right)\left(1-\epsilon_{b}\right) E_{3}^{2}\left(\tau_{0}\right)}
$$

where $E_{b b}$ is the black-body intensity from surface $b$, and $E_{b a}$ is the black-body intensity from surface a.

$$
\begin{aligned}
& \text { Defining } \\
& \chi_{\mathrm{a}}=\frac{\mathrm{J}_{\mathrm{a}}}{\sigma \mathrm{T}_{1}^{4}},
\end{aligned}
$$

and

$$
\chi_{\mathrm{b}}=\frac{\mathrm{J}_{\mathrm{b}}}{\sigma \mathrm{T}_{\mathrm{i}}^{4}}
$$

we obtain

$$
\chi_{a}=\frac{\epsilon_{a} \theta_{a}^{4}+2 \epsilon_{b}\left(1-\epsilon_{a}\right) E_{3}\left(\tau_{0}\right) \theta_{b}^{4}}{1-4\left(1-\epsilon_{a}\right)\left(1-\epsilon_{b}\right) E_{3}^{2}\left(\tau_{0}\right)},
$$


and

$$
\chi_{\mathrm{b}}=\frac{\epsilon_{\mathrm{b}} \theta_{\mathrm{b}}^{4}+2 \epsilon_{\mathrm{a}}\left(1-\epsilon_{\mathrm{b}}\right) \mathrm{E}_{3}\left(\tau_{0}\right) \theta_{\mathrm{a}}^{4}}{1-4\left(1-\epsilon_{\mathrm{a}}\right)\left(1-\epsilon_{\mathrm{b}}\right) \mathrm{E}_{3}^{2}\left(\tau_{0}\right)}
$$

With the above nondimensionalization, Eq. 19 can be expressed as

$$
\frac{\partial \theta}{\partial \zeta}=\frac{1}{\tau_{0}^{2}} \frac{\partial^{2} \theta}{\partial \xi^{2}}+\frac{1}{2 N}\left\{\chi_{a} E_{2}\left(\tau_{0} \xi\right)+\chi_{b} E_{2}\left[\tau_{0}(1-\xi)\right]\right\}
$$

where

$$
\mathrm{N}=\frac{k \kappa}{4 \sigma \mathrm{T}_{\mathrm{i}}^{4}}
$$

The parameter $\mathrm{N}$ determines the relative role of energy transport by conduction to that of radiation. In the limit, as $\mathrm{N}$ approaches infinity, conduction is the dominant mechanism; as $\mathrm{N}$ approaches zero, radiation is the predominant mode of heat transfer. In numerical computations for the steady-state case, Viskanta ${ }^{17}$ formulated the absorbing problem exactly and has determined a "large" $N$ to be 10 and a "small" $N$ to be 0.01 . The partial differential equation (Eq. 29) is seen to be linear. The boundary condi-: tions and initial temperature distribution, specified as $\theta_{i}$, are

$$
\begin{array}{lll}
\zeta=0, & 0 \leq \xi \leq 1, & \theta=\theta_{i} ; \\
\xi=0, & \zeta>0, & \theta=0 ; \\
\xi=1, & \zeta>0, & \theta=1 .
\end{array}
$$

Thus the problem is mathematically well posed. The linear parabolic partial differential equation is second order in space and first order in time. Conditions given by Eqs. 31-33 are sufficient to solve the problem as conceived. Because of the linearity of the governing equation and the boundary condition, the principle of superposition is an obvious technique that is applicable for obtaining an exact analytical solution. This solution is presented in Section III., together with two approximate numerical schemes which are used to obtain solutions for a measure of their utility in the solution of problems of this nature. 


\section{METHODS OF SOLUTION}

This section describes the following three methods for solving the linear partial differential equations for transient heat transfer:

1. Superposition Method.

2. Method of Lines:

3. Lumped-parameter Finite-difference Method.

\section{A. Superposition Method}

An exact analytical solution can be obtained by use of a superposition technique for the solution of Eq. 29 with the initial and nonhomogeneous boundary conditions given by. Eqs. 31-33. Thus, assuming that $\theta$ can be written as

$$
\theta(\xi, \zeta)=U(\xi, \zeta .)+V(\xi)
$$

the following systems of equations result and can be independently solved. These systems are an initial condition problem; i.e.,

$$
\begin{aligned}
& \frac{\partial U}{\partial \zeta}=\frac{1}{T_{0}^{2}} \frac{\partial^{2} U}{\partial \xi^{2}} \\
& U(\xi, 0)=1-V(\xi) \\
& U(0, \zeta)=0 \\
& U(1, \zeta)=0
\end{aligned}
$$

and an ordinary differential equation that accounts for the inhomogeneous boundary conditions,

$$
\begin{aligned}
& \frac{\mathrm{d}^{2} \mathrm{~V}}{\mathrm{~d} \xi^{2}}+\frac{\tau_{0}^{2}}{2 \mathrm{~N}}\left\{\chi_{\mathrm{a}} \mathrm{E}_{2}\left(\tau_{0} \xi\right)+\chi_{\mathrm{b} \mathrm{E}_{2}}\left[\tau_{0}(1-\xi)\right]\right\}=0 \\
& \mathrm{~V}(0)=\theta_{\mathrm{a}}=0
\end{aligned}
$$

and

$$
\mathrm{V}(1)=\theta_{\mathrm{b}}=1
$$

The solution to the ordinary linear differential equation (Eq. 39) with the boundary conditions given by Eqs. 40 and 41 is 


$$
\begin{aligned}
V(\xi)= & -\frac{1}{2 N}\left\{\chi_{a} E_{4}\left(\tau_{0} \xi\right)+\chi_{b} E_{4}\left[\tau_{0}(1-\xi)\right]\right. \\
& \vdots \\
- & \left.\left(\chi_{a}-\chi_{b}\right)\left[E_{4}\left(\tau_{0}\right)-\frac{1}{3}\right] \xi-\left[\frac{\chi_{a}}{3}+\chi_{b} E_{4}\left(\tau_{0}\right)\right]\right\}
\end{aligned}
$$

In general, in the solution of linear parabolic partial differential equations, two separate series can be obtained, one a.series of trigonometric functions (for slab geometry), the other a series of error functions. The trigonometric series is rapidly convergent for long times, whereas the error-function series is rapidly convergent for short times. Thus, in the solution of Eq. 35, we will obtain a long-time solution analytically, and when numerically evaluating the resultant series solution, we will compute solutions for as small times as are numerically practical.

What remains then, is to obtain a solution to Eq. 35 with the conditions of Eqs. 36-38: By separation of variables, the formal solution can be directly obtained and may be written as

$$
\dot{U}(\xi, \zeta)=\sum_{k=1}^{\infty} A_{k} \mathrm{e}^{-\left(\mathrm{k} \pi / T_{0}\right)^{2} \zeta} \sin (\mathrm{k} \pi \xi)
$$

where

$$
\begin{aligned}
A_{k}= & \frac{1}{2} \int_{0}^{1}\left\{\left[1-\frac{\chi_{a}}{6 N}-\frac{\chi_{b}}{2 N} E_{4}\left(\tau_{0}\right)\right] \sin (k \pi \xi)\right. \\
\therefore \quad & \frac{1}{2 N}\left(\chi_{a}-\chi_{b}\right)\left[E_{4}\left(\tau_{0}\right)-\frac{1}{3}\right] \xi \sin (k \pi \xi) \\
& \left.+\frac{\chi_{a}}{2 N} E_{4}\left(\tau_{0} \xi\right) \sin (k \pi \xi)+\frac{\chi_{b}}{2 N} E_{4}\left[\tau_{0}(1-\xi)\right] \sin (k \pi \xi)\right\} d \xi \\
& +\frac{1}{2} \int_{0}^{1} \xi \sin (k \pi \xi) d \xi .
\end{aligned}
$$

The last two terms on the right-hand side of Eq. 44 are integrated in Appendix B. The results are 


$$
\begin{aligned}
2 \mathrm{~A}_{\mathrm{k}}= & {\left[1-\frac{\chi_{\mathrm{a}}}{6 \mathrm{~N}}-\frac{\chi_{\mathrm{b}}}{2 \mathrm{~N}} \mathrm{E}_{4}\left(\tau_{0}\right)\right]\left[\frac{1-(-1)^{\mathrm{k}}}{\mathrm{k} \pi}\right]-\frac{(-1)^{\mathrm{k}}}{\mathrm{k} \pi} \frac{1}{2 \mathrm{~N}}\left[\chi_{\mathrm{a}}-\chi_{\mathrm{b}}\right] } \\
& {\left[\frac{\mathrm{E}_{4}\left(\tau_{0}\right)(-1)^{\mathrm{k}}-\frac{1}{3}}{\mathrm{k} \pi}\right](-1)^{\mathrm{k}}+\left[( \frac { \tau _ { 0 } } { \mathrm { k } \pi } ) ^ { 3 } \left[\frac{1}{\mathrm{k} \pi}\left(\mathrm{si} \frac{\mathrm{k} \pi}{\tau_{0}}+\frac{\pi}{2}\right)+\frac{\frac{1}{3}-\mathrm{E}_{4}\left(\tau_{0}\right)(-1)^{\mathrm{k}}}{\mathrm{k} \pi}\right.\right.} \\
& +\left(\frac{\tau_{0}}{\mathrm{k} \pi}\right)^{2}\left[\frac{E_{2}\left(\tau_{0}\right)(-1)^{\mathrm{k}}-1}{\mathrm{k} \pi}\right]+\sum_{\ell=1}^{\infty} \frac{(-1)^{\ell-1} \tau_{0}^{\ell}}{\ell \cdot \ell !}\left\{\frac { ( - 1 ) ^ { \mathrm { k } } } { \mathrm { k } ^ { \ell + 1 } } \sum _ { \mathrm { m } = 0 } ^ { \mathrm { I } ( \frac { \ell - 1 } { 2 } ) } \left[\frac{(-1)^{\mathrm{m}} \ell !}{(\ell-2 \mathrm{~m}-1) !}(\mathrm{k} \pi)^{\ell-2 \mathrm{~m}-1}\right.\right. \\
+ & \left.\left.\left.(-1)^{\mathrm{I}^{\prime}(\ell-1) / 2} \frac{2 \mathrm{I}\left(\frac{\ell-1}{2}\right)-\ell}{\mathrm{k}^{\ell+1}}\right]\right\}\right]\left[\frac{\chi_{\mathrm{a}}}{2 \mathrm{~N} !}+\frac{(-1)^{\mathrm{k}-1} \cdot \chi_{\mathrm{b}}}{2 \mathrm{~N}}\right]
\end{aligned}
$$

The general solution is then:

$$
\begin{aligned}
\theta(\xi, \zeta)= & \xi-\frac{1}{2 \mathrm{~N}}\left\{\chi_{\mathrm{a}} \mathrm{E}_{4}\left(\tau_{0} \xi\right)+\chi_{\mathrm{b}} \mathrm{E}_{4}\left[\tau_{0}(1-\xi)\right]\right. \\
& \left.-\left(\chi_{\mathrm{a}}-\chi_{\mathrm{b}}\right)\left[\mathrm{E}_{4}\left(\tau_{0}\right)-\frac{1}{3}\right] \xi-\left[\frac{\chi_{\mathrm{a}}}{3}+\chi_{\mathrm{b}} \mathrm{E}_{4}\left(\tau_{0}\right)\right]\right\} \\
& +\sum_{\mathrm{k}=1}^{\infty} \mathrm{A}_{\mathrm{k}} \mathrm{e}^{-\left(\mathrm{k} \pi / \tau_{0}\right)^{2}} \zeta \sin (\mathrm{k} \pi \xi)
\end{aligned}
$$

where $A_{k}$ is given by Eq. 45. Equation 46 was evaluated on an IBM, 360-50 machine in double-precision arithmetic using a convergence criterion for the above series of $\leq 10^{-7}$. Because of the restrictive convergence used, run times were fairly lengthy ( $\approx 15 \mathrm{~min}$ ), but the accuracy is assumed to be good.

This method used for the solution of the proposed problem is considered to be exact. In Sections $B$ and $C$ below, two finite-difference methods are used to solve the same problem. The purpose of the approximate solutions is to check the usefulness of these methods to determine whether they would be useful when the solution could not be determined in an exact fashion. One difficulty stands out when attempting to apply these approximate techniques to a more exact model. This is the fact that a more exact model would probably contain highly nonlinear terms for which the convergence criterion herein obtained would no longer be useful. The 
handling of nonlinear terms in any finite-difference scheme becomes an art unto the problem at hand, rather than a science, but we propose that in the absence of other information, the values obtained here could be used as a starting point.

\section{B. Method of Lines}

The second technique used for the solution of the problem is the method of lines ${ }^{18}$ (often referred to in the English literature as the differential-difference method). Tien ${ }^{19}$ uses essentially this method in the solution of steady-state conduction problems and calls it the strip method. Perhaps one of the first to use it in the English literature is Eyres et al ${ }^{20}$ for the reduction of partial differential equations to sets of ordinary differential equations. The Russian literature often refers to this method in connection with Dorodnitsyn, ${ }^{21}$ who was apparently the first to use this scheme. The advantage of this approach for linear problems is that analytical solutions to the resulting ordinary differential equations may be possible. In addition, it is often possible to estimate the error introduced by the approximation and, if necessary, apply an approximate correction for it.

For the one-dimensional transient-heat conduction equation, there are two alternative methods of applying this technique. The first method is to replace the time derivative by the temperature change during a finite time interval. Such an approximation has been examined for accuracy ${ }^{22,23}$ and has been applied successfully to an internal-heat-generation problem. ${ }^{19}$ However, whether the equation is linear or not, the problem in the space dimension will be a two-point boundary-value problem, which will be more difficult to solve numerically.

A simpler and more practical treatment is to replace the spatial. derivative by a corresponding finite difference. ${ }^{24}$ This method will be presented here for the problem given by Eq. 27 and the boundary conditions given by Eq. 29.

We will replace the spatial derivative by difference equations derivable from Taylor series in the following manner: Let $\theta_{\xi}(\zeta)$ be the value of $\theta$ at $\xi=n \Delta \xi$ for all time $\zeta$, and

$$
\Delta \xi=\frac{1}{H} .
$$

where $H$ is the number of intervals into which $0 \leq \xi \leq 1$ is divided. The Taylor-series expansions for $\theta_{n+1}$ and $\theta_{n-1}$ to order $(\Delta \xi)^{3}$ are

$$
\theta_{\mathrm{n}+1}=\theta_{\mathrm{n}}+\frac{\Delta \xi}{1 !} \frac{\partial \theta_{\mathrm{n}}}{\partial \xi}+\frac{(\Delta \xi)^{2}}{2 !} \frac{\partial^{2} \theta_{\mathrm{n}}}{\partial \xi^{2}}+\ldots
$$


and

$$
\theta_{\mathrm{n}-1}=\theta_{\mathrm{n}}-\frac{\Delta \xi}{1 !} \frac{\partial \theta_{\mathrm{n}}}{\partial \xi}+\frac{(\Delta \xi)^{2}}{2 !} \frac{\partial^{2} \theta}{\partial \xi^{2}}-\cdots
$$

Subtracting and solving for $\partial \theta_{n} / \partial \xi$, we obtain

$$
\frac{\partial \theta_{n}}{\partial \xi}=\frac{\theta_{n+1}-\theta_{n-1}}{2 \Delta \xi}, \quad l \leq n \leq H-1 .
$$

Adding and solving for $\partial^{2} \theta / \partial \xi^{2}$, we obtain

$$
\frac{\partial^{2} \theta_{n}}{\partial \xi^{2}} \dot{=} \frac{\theta_{\mathrm{n}+1}-2 \theta_{\mathrm{n}}+\theta_{\mathrm{n}-1}}{(\Delta \xi)^{2}}
$$

From the Taylor-series expansion we see that this is equivalent to replacing the temperature distribution by a cubic between $\xi_{\mathrm{n}}$ and $\xi_{\mathrm{n}+\mathrm{l}}$. The errors introduced by this approximation can be decreased by decreasing $\Delta \xi$, that is, by increasing the number of intervals into which the thickness of the slab is divided. One of the primary reasons for this work is to determine for this problem the smallest value of $H$ required to give adequate accuracy so that the method may be used to solve more complex nonlinear problems with some confidence.

Using the above approximation in the partial differential equation, we obtain $\mathrm{H}+1$ ordinary differential equations for $\theta_{\mathbf{n}}(\zeta), \mathrm{n}=0,1, \ldots, \mathrm{H}$ in one independent variable $\zeta$ :

$$
\frac{\mathrm{d} \theta_{\mathrm{n}}}{\mathrm{d} \zeta}=\frac{1}{\tau_{0}^{2}}\left\{\frac{\partial^{2} \theta_{\mathrm{n}}}{\partial \xi^{2}}\right\}+\frac{1}{2 \mathrm{~N}}\left\{\chi_{\mathrm{a}}^{4} \mathrm{E}_{4}\left(\tau_{0} \xi\right)+\chi_{\mathrm{b}} \mathrm{E}_{\mathrm{b}}\left[\tau_{0}(1-\xi)\right]\right\} .
$$

In the above formulation, $\theta_{\mathrm{a}}$ and $\theta_{\mathrm{b}}$ are the surface temperatures determined as boundary conditions given by Eqs. 32 and 33 . Thus

$$
\frac{\mathrm{d} \theta_{0}}{\mathrm{~d} \tau}=0
$$

With the above we determine that the problem is well posed in a mathematical sense. What remains is to solve the remaining $\mathrm{H}+1$ coupled ordinary differential equations for the initial conditions given by Eq. 31 . This was done by use of a fifth-order Runge-Kutta forward-integration scheme on an IBM 360-50 computer. The time-step sizes used were $10^{-3}$ and $10^{-4}$; a comparison of the two solutions for $\mathrm{H}=30$ showed them to be identical out to the seventh significant figure. For this reason the integration was assumed correct to the order of the scheme. 
Sarmin and Chudov ${ }^{37}$ investigated the stability of the computation of the system of ordinary differential equations obtained from the method of lines. One of the problems under investigation is the diffusion equation without a heat-source term. Addition of the apparent heat-source term does not alter the analysis appreciably. Fortunately the analys is is given for a fourth-order Runge-Kutta forward-integration scheme. An analysis similar to Sarmin and Chudov's demonstrated that for the discretization parameters chosen the necessary and sufficient conditions for stability were satisfied in the computations performed.

Kostynkovich ${ }^{23}$ has done some work on the convergence of this method. His analysis shows that for problems such as the ones posed here, we find convergence to the exact solution for "large" $\mathrm{H}$ (in present terminology). In actual runs, convergence to the analytical solution was obtained for large times by dividing the $\xi$ interval into seven equal increments. For "small" time, the closer we attempt to get to a step function in temperature, the more difficult the solution is to obtain. Forty increments were used with good accuracy ( $4 \%$, based on the exact solution) throughout the interval.

\section{Lumped-parameter Finite-difference Method}

A third method ${ }^{25}$ used for the solution of the problem is to obtain confidence in the techniques that hopefully can be extrapolated to the non-

- linear cases, which are obvious extensions of the current problem. This solution is a lumped-parameter finite-difference technique, assembling a heat balance in implicit form for each node, and applying the Gauss-Seidel ${ }^{26}$ method to the resultant system of $n$ algebraic equations relating the $n$ unknown node temperatures. An iteration scheme is used for each specified time step during the transient until temperatures at node centers have been computed for all the time steps with some preselected error limit.

Because the method chosen is based upon an implicit finite-difference scheme, the solution is unconditionally stable. ${ }^{27}$ In fact, the steady-state solution may be easily obtained by setting the time increment to an excessively large value. However, this requires a large number of iterations for convergence. On the other hand, with the extreme speed of the machine used (CDC-3600) and the ability of the program to rapidly solve the system of algebraic equations that result for a single time step, it is feasible to .take sufficiently small time steps to reduce truncation errors inherently present in finite-difference schemes to well below $2 \%$.

A maximum of 60 iterations was used as an upper limit. This limit, if attained, would cause a statement to be printed notifying the user of this fact and then to permit the machine to proceed to the next time step. To attain steady-state conditions, a different iteration limit must be used after the limit specified for the last time step. 
One important result of the computations is that during periods of rapid temperature change, small time steps must be used or truncation errors will be excessive. After the period of rapid change is over, the time steps should be increased in a geometric progression.

The analysis used was of the standard form given in the literature on finite differences ${ }^{25,27}$

The first law requires that

$$
\text { Q absorbed }=Q_{\text {in }}-Q_{\text {out }} \text {, }
$$

where the last two terms contain the conduction portion of the energy equation Eq. 29. We can represent Eq. 52 by

$$
D_{i}\left(\theta_{i}^{\prime}-\theta_{i}\right)=\sum_{\substack{j=1 \\ j \neq i}}^{n}\left[D_{i j}\left(\frac{\theta_{j}+\theta_{j}^{\prime}}{2}-\frac{\dot{\theta}_{i}+\dot{\theta}_{i}^{\prime}}{2}\right)\right]+Q_{i}
$$

where $Q_{i}$ is the internal-heat-generation rate, which may in general be a function of space and/or time,

$$
D_{i}=\xi_{i} \frac{3600}{\Delta \zeta}
$$

and

$$
D_{i j}=\text { Area of face of node } i j
$$

Expanding and collecting terms in the unknown temperature on the left, we obtain

$$
\begin{gathered}
D_{i, 1} \theta_{1}^{\prime}+D_{i, 2} \theta_{2}^{\prime}+\ldots-\left[\sum_{\substack{j \neq 1 \\
j \neq i}}^{n} D_{i j}+2 D_{i}\right] \theta_{i}^{\prime} \\
+\ldots-D_{i, n} \theta_{n}^{\prime}=\theta_{i}\left[\sum_{\substack{j \neq 1 \\
j \neq i}}^{n} D_{i j}-2 D_{i}\right]-\sum_{\substack{j=1 \\
j \neq i}}^{n} D_{i j} \theta_{j}-2 Q_{i}
\end{gathered}
$$

If the coefficient of the term in $\theta_{i}$ is defined as $D_{i i}$, where 


$$
D_{i i}=-\left[\sum_{\substack{j=1 \\ j \neq i}}^{n} D_{i j}+2 D_{i}\right]
$$

and $\bar{\Phi}$ represents the right-hand side of Eq. 54 , then for $i=1,2, \ldots, n$, the first law stated for $n$ segments results in a set of simultaneous equations in $n$ unknowns, $\bar{\theta}_{i}^{\prime}$, expressed as

$$
\overline{\mathrm{D}} \bar{\theta}^{\prime}=\bar{\Phi}
$$

where $\bar{\theta}^{\prime}$ is the solution vector of temperatures, $\bar{\Phi}$ is the vector of constants, and $\overline{\bar{D}}$ is the coefficient matrix.

The diagonal of $\bar{D}, D_{i j}$, is strong, in particular when the term $D_{i}$ is large (which is true when the time interval is small) and $D$ has many locations with small or zero values. For any $i$, a maximum of seven $D_{i j}$ terms are nonzero and a minimum of one $D_{i j}$ term is nonzero.

For the solution of the simultaneous equations, the coefficient matrix is first stored in $n \times 7$ core locations. In this scheme, the iterates to the solution vector $\bar{\theta}_{i}^{\prime}$ are arrived at by transforming Eq. 55 to the form.

$$
\bar{\theta}^{\prime}=\bar{B} \bar{\theta}^{\prime}+F \text {. }
$$

To do this, place all the terms, except those on the diagonal, on the right side, and then divide them by the diagonal coefficient. Thus the elements of the matrix $\bar{B}$ become

$$
\begin{aligned}
& b_{i j}=-\frac{D_{i j}}{D_{i i}}, \quad j \neq i ; \\
& b_{i i}=0, . \quad j=i .
\end{aligned}
$$

The elements of $\overline{\mathrm{F}}$ are defined by

$$
f_{i}=\frac{S_{i}}{D_{i i}}
$$

At this point, we use the formula

$$
\theta_{i}^{\prime}(k+1)=\sum_{j=1}^{i-1} b_{i j} \theta_{j}^{\prime}(k+1)+\sum_{j=i+1}^{\dot{n}} b_{i j} \theta_{j}^{\prime}(k)+f_{i}
$$


where the superscript $k$ denotes the order of the iteration. With the above procedure, we can specify any convergence criterion for a particular time step.

\section{RESULTS AND DISCUSSION}

The results obtained by the three methods used are given in Figs. 215. Boundary conditions imposed and parameters chosen as being representative are noted on several figures. In general, the parameter $\mathrm{N}$, which represents the ratio of the relative importance of hëat transfer by conduction to that by radiation, is chosen to be $10,1.0,0.1$, and:0.01, while the optical thicknesses considered are $0.1,0.5,1.0$, and 10 . The dimensionless temperature, $\theta(\xi, \xi)$, has the initial condition 0.111 and the boundary conditions 0 and 1.0 .

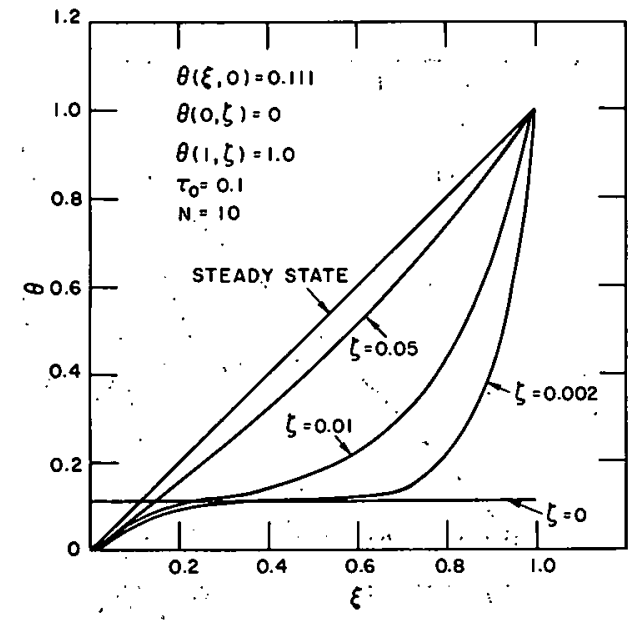

$112-9188$

Fig. 2. Solutions of Eq. 29 for $\mathrm{N}=10, \therefore \tau_{0}=0 . \dot{1}$

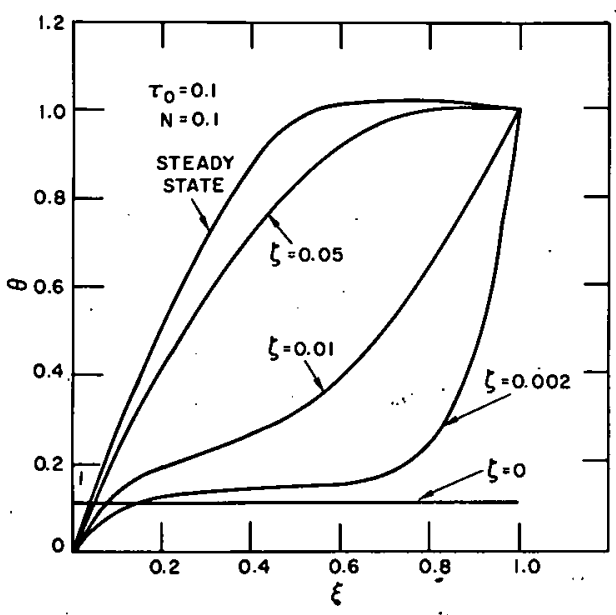

$112-9190$

Fig. 4. Solutions for Éq. 29 for $\mathrm{N}=0.1, \tau_{0}=0.1$

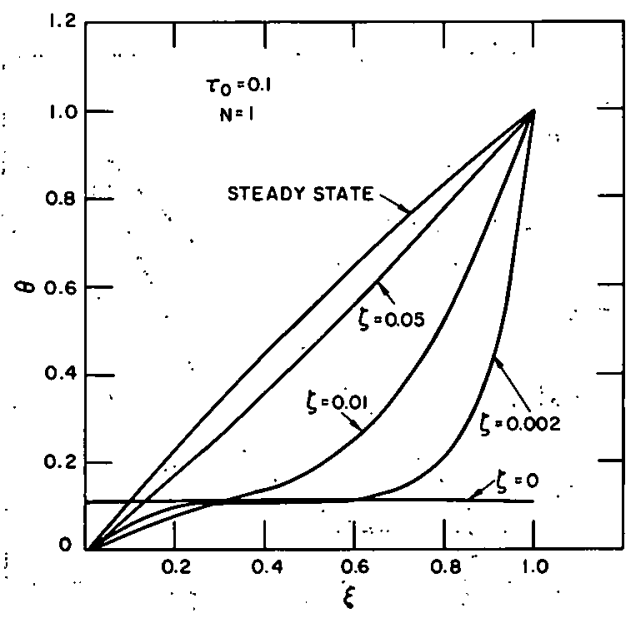

$112-9189$

Fig. 3. Solutions of Eq. 29 for

$\because \cdots, \mathrm{N}=1, \tau_{0}=0.1$

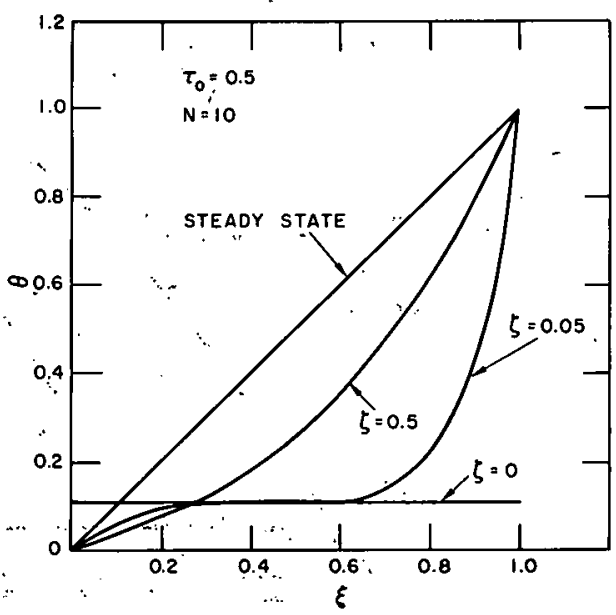

$112-9191$

Fig. 5. Solutions for Eq. 29 for $\mathrm{N}=10, \tau_{0}=0.5$ 


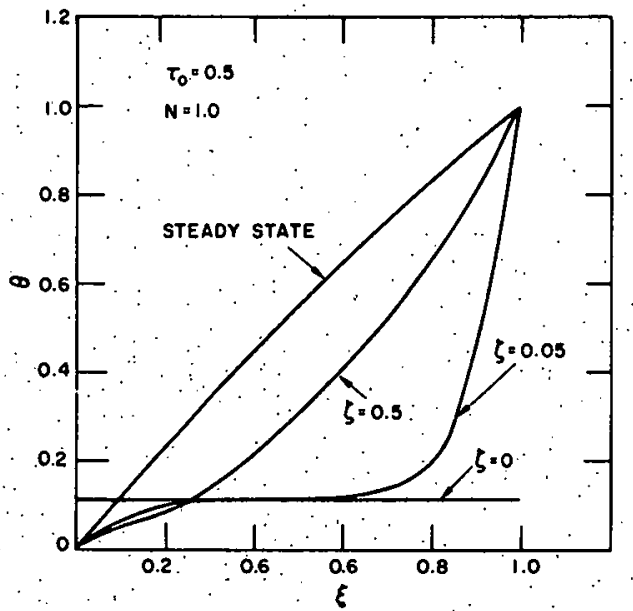

$112-9192$

Fig. 6. Solutions of Eq: 29 for

$$
\mathrm{N}=1, \tau_{0}=0.5
$$

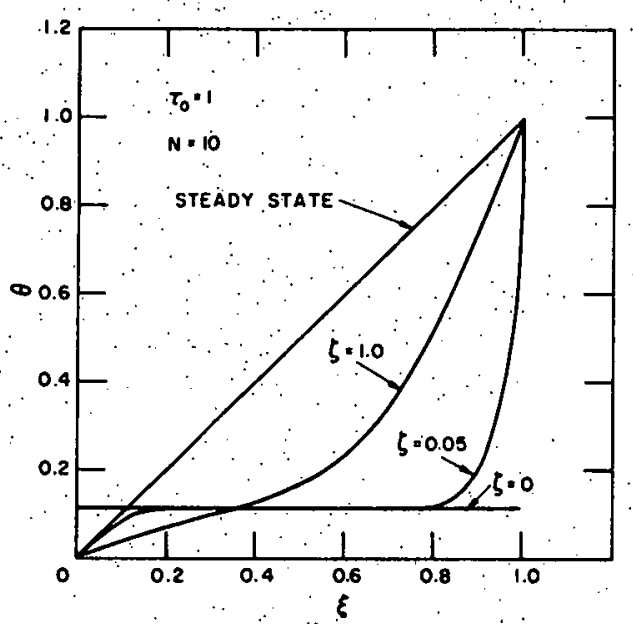

$112-9194$

Fig. 8. Solutions of Eq. 29 for $\mathrm{N}=10, \tau_{0}=1$

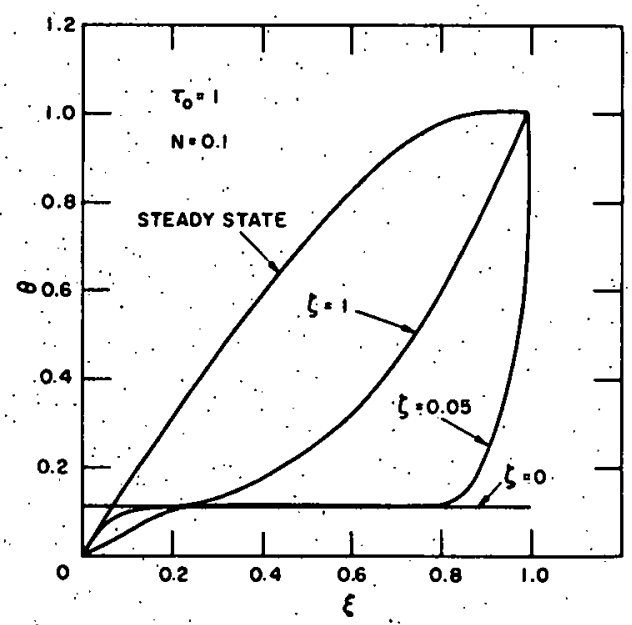

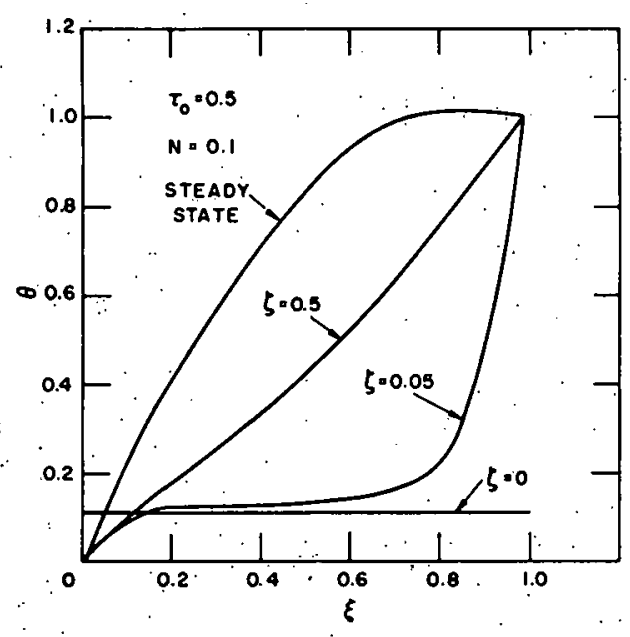

$112-9193$

Fig. 7. Solutions of Eq. 29 for $\mathrm{N}=0.1, \tau_{0}=0.5$

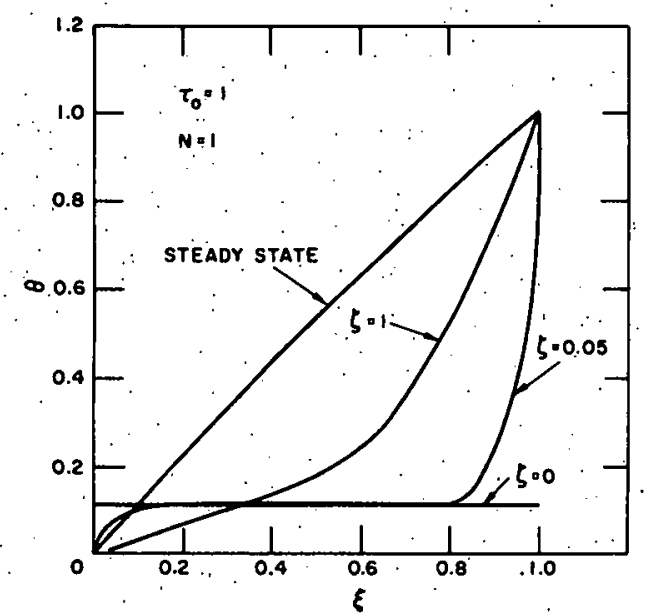

112-9195

Fig. 9. Solutions of Eq. 29 for $\mathrm{N}=1, \tau_{0}=1$

Fig. 10

Solutions of Eq. 29 for $\mathrm{N}=0.1, \mathrm{~T}_{0}=1$ 


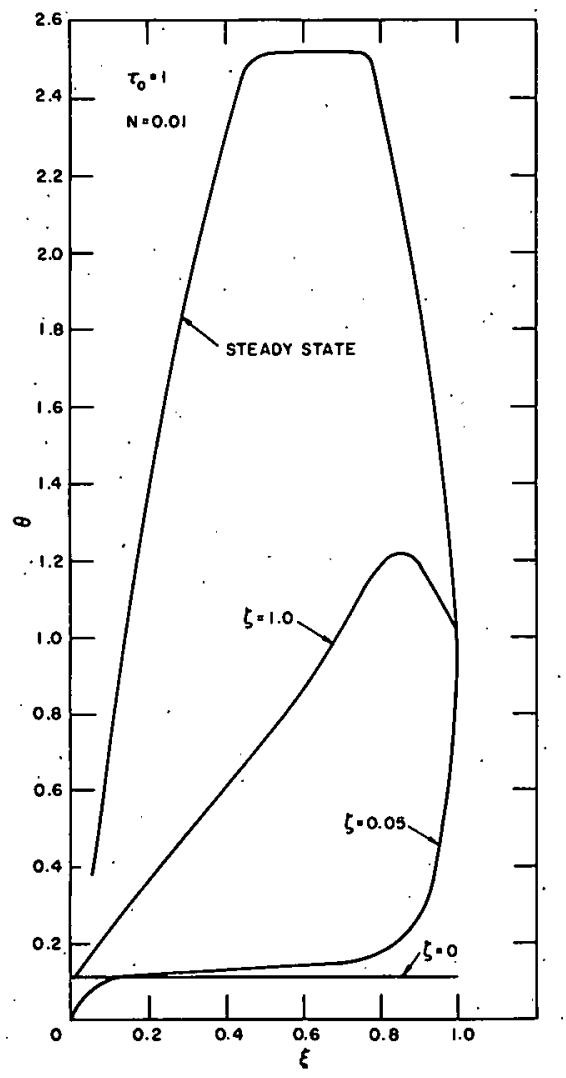

112-9197

Fig. 11. Solutions of Eq. 29 for $\mathrm{N}=0.01, \tau_{0}=1$

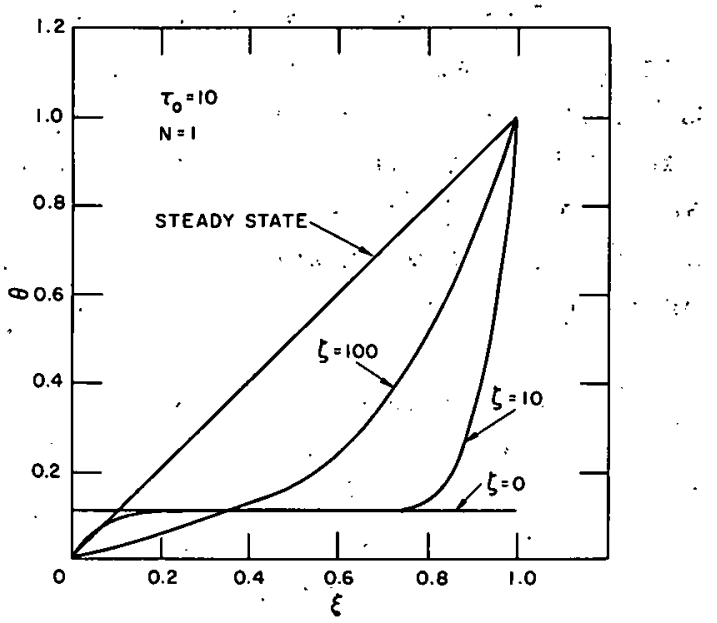

112-9199

Fig. 13. Solutions of Eq. 29 for $\mathrm{N}=1, \tau_{0}=10$

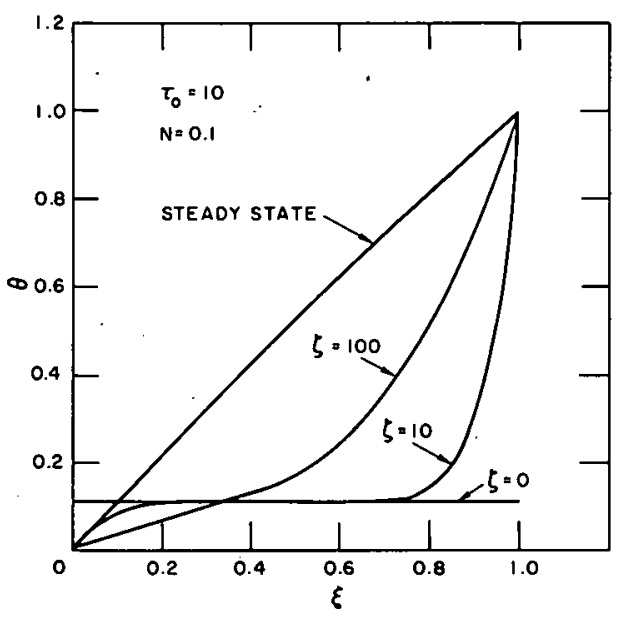

$112-9200$

Fig. 14. Solutions of Eq. 29 for $\mathrm{N}=0.1, \tau_{0}=10$

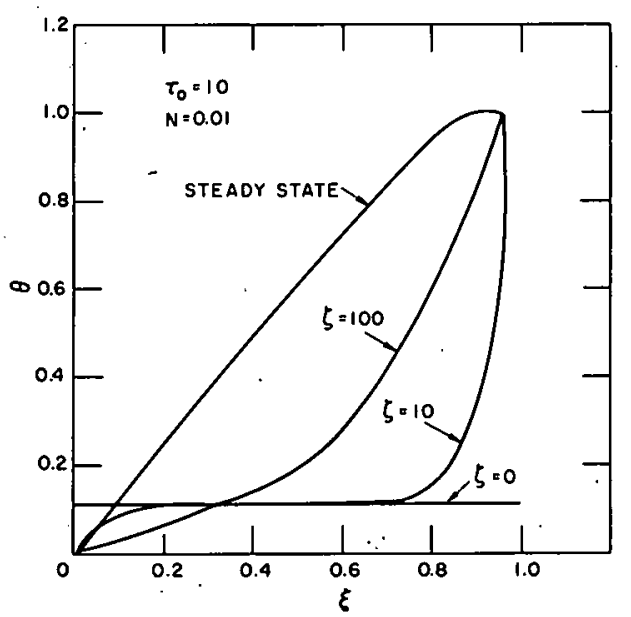

112-9201

Fig. 15. Solutions of Eq. 29 for
. $\mathrm{N}=0.01, \tau_{0}=10$
Fig. 12. Solutions of Eq. 29 for $\mathrm{N}=10, \tau_{0}=10$ 
Figures 3 and 4 illustrate the effect of the absorption of radiant energy for an optical thickness of 0.1 . In general, for arbitrary optical thickness the effect of absorption is to increase the local enthalpy level within the medium. The pure-conduction solutions, that is, with no absorption, for the same boundary and initial conditions agree to within six decimals with the solutions obtained for $N=10$ for all values of the optical thickness.

In all cases, changing the value of $\mathrm{N}$ from ten to one had but a small effect on the solutions. Correspondingly, changing $\mathrm{N}$ from one to one-tenth had a more appreciable effect on the results for a given optical thickness. Results for $N=0.01$ when $\tau_{0}=0.1$ and 0.5 are not presented for all cases because of the excessively large temperatures attained near the "hot" $(\theta=5.0)$ wall. This is due to the intense absorption of the radiation, with

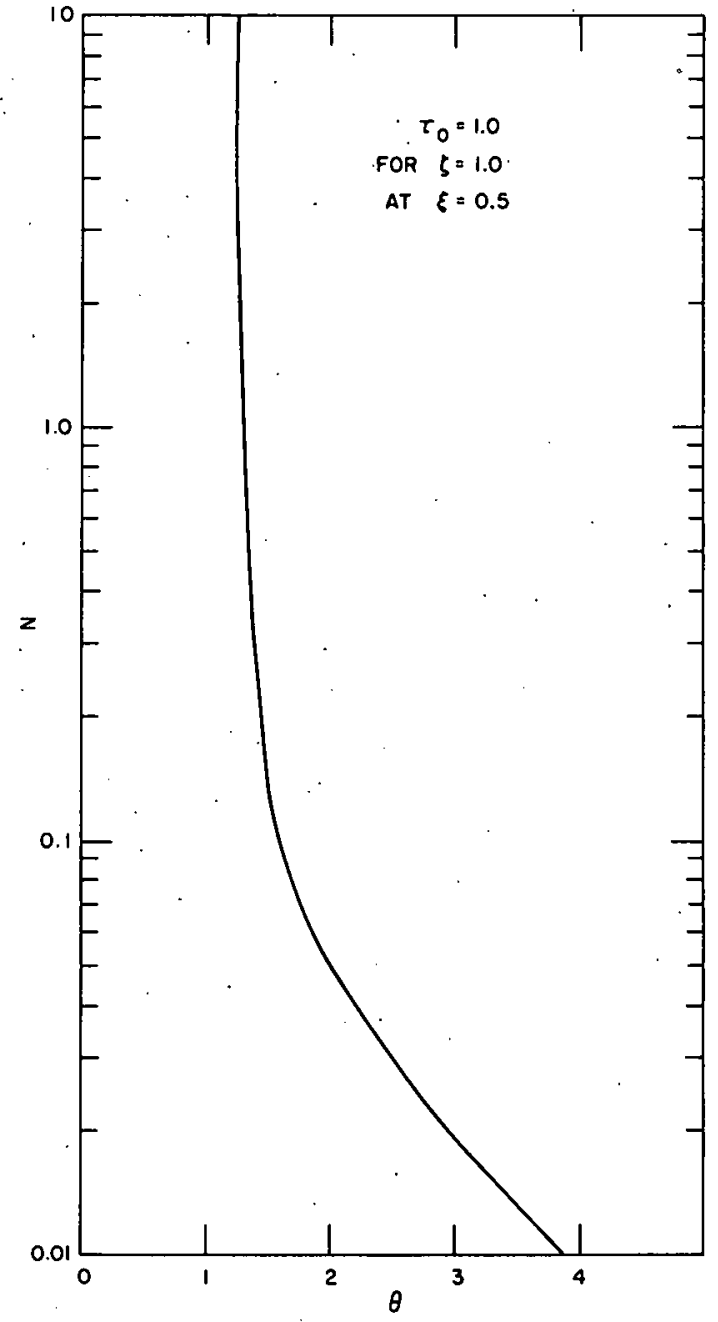

$112-9202$

Fig. 16. Effect of Parameter $N$ on Temperature at $\xi=0.5$ and $R=1.0$ when $\tau_{0}=1.0$ conduction being the only mechanism available for dissipation of the energy. Although the radiant energy emitted by the medium is neglected, an energy balance is still maintained; enthalpy and other recognized forms of energy are undiminished by the emission of radiation. The model does not permit the medium to emit radiation, but in practice radiation would actually be emitted. Particular note of this fact can be made by referring to Figs. 4 and 11 where the steady-state profile is definitely unbalanced because the radiation acts as a heat source. This situation is not compatible with the physical model under consideration. The emission that is neglected would tend to decrease the temperature below that of the wall. On the other hand, for a large value of optical thickness, as demonstrated by Fig. 15, we see that for $N=0.01$ the heat-source effect is much smaller.

For increasing values of the optical thickness, the general effect is to decrease the time response of the medium, that is, to increase the time required to attain steady state. Figure 16 shows the effect of the parameter $\mathrm{N}$ on the dimensionless temperature to reach a preset value of the dimensionless time 
(i.e., $\zeta=1.0$ ) and space (i.e., $\xi=0.5$ ) when the total optical thickness is unity.. The effect of decreasing $\mathrm{N}$ is to increase the rate of heat transfer, as would be expected with an increase in the importance of radiation. $\because: \because$

The results presented are affected by the as sumptions: made regarding the mechanisms involved; however, the solution to the problem as posed is exact in an analytical sense. A comparison of the two numerical methods used in the solution of the governing equations with the exact analytical solution results in no greater than $0.05 \%$ error, except during the initial $\left(\zeta \leq 10^{-5}\right)$ stages of the transient, where it is extremely difficult to accurately approximate the step function that is a result of the suddenly changed boundary conditions. The results as given were extremely encouraging with regard to the use of these numerical methods for the extension of this problem to the nonlinear absorbing and emitting case and even to the more realistic nongray situation.

Results obtained for different boundary conditions and initial conditions showed the same trends as those álready discussed. Decreasing the wall emis sivities to $0.1,0.1 ; 0.1,0.7$; and $0.5,0.9$ decreased the heat-source effect to a degree related to the value of the emissivity of the wall with the highest temperature. No other obvious effects were noted, which is expected due to the linearity of the governing equation, boundary conditions, and initial condition.

Thus we see that the absorption by a relatively low-temperature medium does affect the time response and temperature distribution. It is expected that the results of the approximate analysis will retain the significant qualitative aspects of the actual physical behavior. The solution of combined radiation and conduction problems may be obtained with good accuracy using either the method of lines or the implicit finite-difference approach. Hopefully the nonlinearities introduced by a rigorous treatment of the problem will not affect the stability or convergence of the schemes proposed.

\section{CONCLUSIONS AND RECOMMENDATIONS}

Transient heat transfer by combined conduction and radiation in a nonscattering, nonemitting, absorbing, gray medium confined between two infinite parallel plates has been studied. The governing linear partial differential equation is developed from first principles. Solutions are obtained by an exact analytical method and compared with the method of lines and an implicit finite-difference technique to obtain confidence in numerical methods that obviously are necessary for the solution of a rigorously formulated problem. 
The solutions are presented for values of the parameter $\mathrm{N}$ of 1.0 , $1: 0,0.1$, and 0.01 with values of optical thickness of $10,1.0,0.5$, and 0.1 . The results are characterized in terms of dimensionless time, dimensionless temperature, and the above two parameters. Temperature distributions at steady state for $\mathrm{N}=10$ are linear for some cases, as is characteristic of pure conduction without heat sources present. For $\mathrm{N}=10,1.0,0.1$, and 0.01 , and $\tau_{0}=10$, the evidence of radiation as a diffusion phenomenon is readily apparent. For appreciable energy transfer by radiation in comparison with conduction $(\mathrm{N}=0.01)$, the model is invalid for small or intermediate values of optical thickness as the steady state is approached.

Because of the linear nature of the problem, convergence, stability, and uniqueness present no obstacle in obtaining a solution. The two numerical techniques used appear to be acceptable for use in more sophisticated approaches to the problem. In practice, the method of lines is easier than the implicit finite-difference formulation. Once the basic program has been written, however, the two methods may be compared from a stability standpoint. The finite-difference formulation is implicit in form and thus is inherently stable for linear problems and normally stable for nonlinear problems. On the other hand, the method of lines is usually termed an explicit method, which is often found to be unstable. Thus the method of lines requires some discretion on the part of the user with regard to stability.

In the problem solved here, there is no indication regarding a like problem existing on the convergence of the method.

A simple model has been developed for combined conduction and radiative transfer between two parallel plates. The two parameters in the model can be evaluated given experimental and/or analytical information regarding the media. The effect of radiation absorption as a heat source is noted, and information on its importance as a contributing mechanism is obtained. Results obtained by numerical means compares.very well with the exact analytical solution. Tables I and II compare the results based on the exact method, the method of lines, and the lumped-parameter approach. These representative results demonstrate the agreement of the three methods. 
TABLE I. Comparison of Numerical Values Obtained by the Three Methods at Discrete Values of the Space Variable for $\zeta=0.004$ and for. Values of the Parameters $N=0.1$ and $\tau_{0}=0.1$

\begin{tabular}{cccc}
\hline $\begin{array}{c}\text { Space } \\
\text { Variable, } \\
\xi\end{array}$ & \multicolumn{2}{c}{ Temperature, $\theta$} \\
\cline { 2 - 4 } & $\begin{array}{c}\text { Exact } \\
\text { Method }\end{array}$ & $\begin{array}{c}\text { Method of } \\
\text { Lines }\end{array}$ & $\begin{array}{c}\text { Lumped-parametèr } \\
\text { Method }\end{array}$ \\
\hline 0.00 & 0.0000 & 0.0000 & 0.0000 \\
0.05 & 0.3932 & 0.3931 & 0.3932 \\
0.15 & 0.2398 & 0.2397 & 0.2398 \\
0.25 & 0.1588 & 0.1587 & 0.1588 \\
0.35 & 0.1235 & 0.1234 & 0.1234 \\
0.45 & 0.1121 & 0.1120 & 0.1120 \\
0.55 & 0.1170 & 0.1169 & 0.1169 \\
0.65 & 0.1466 & 0.1465 & 0.1465 \\
0.75 & 0.2289 & 0.2288 & 0.2289 \\
0.85 & 0.4134 & 0.4132 & 0.7133 \\
0.95 & 0.7598 & 0.7596 & 1.0000 \\
1.00 & 1.0000 & 1.0000 & \\
\hline
\end{tabular}

TABLE II. Comparison of Numerical Values Obtained by the Three Methods at Discrete Values of the Space Variable for $\zeta=0.04$ and for Values of the Parameters $N=0.1$ and $\tau_{0}=0.1$

\begin{tabular}{cccc}
\hline $\begin{array}{c}\text { Space } \\
\text { Variable, } \\
\xi\end{array}$ & $\begin{array}{c}\text { Temperature, } \theta \\
\text { Method }\end{array}$ & $\begin{array}{c}\text { Method of } \\
\text { Lines }\end{array}$ & $\begin{array}{c}\text { Lumped-parameter } \\
\text { Method }\end{array}$ \\
\hline 0.00 & 0.0 .0000 & 0.00000 & 0.00000 \\
0.05 & 0.48453 & 0.48451 & 0.48452 \\
0.15 & 0.45959 & 0.45956 & 0.45957 \\
0.25 & 0.44693 & 0.44690 & 0.44690 \\
0.35 & 0.45219 & 0.45215 & 0.45214 \\
0.45 & 0.47937 & 0.47938 & 0.47934 \\
0.55 & 0.53072 & 0.53075 & 0.53071 \\
0.65 & 0.60591 & 0.60594 & 0.60591 \\
0.75 & 0.70235 & 0.70236 & 0.81496 \\
0.85 & 0.81494 & 0.81498 & 0.93735 \\
0.95 & 0.93737 & 0.93734 & 1.00000 \\
1.00 & 1.00000 & 1.00000 & \\
\hline
\end{tabular}


APPENDIX A

Absorption Analysis

To analyze the importance of radiative energy transfer as a con-" tributing mechanism to total energy transfer, we must reduce the available experimental information on the absorption cross section for sodium vapor to the Planck-mean absorption coefficient. At present the Planck-mean absorption coefficient appears to be the most meaningful average to use. for the mean extinction coefficient. ${ }^{31}$ Knowing the Planck-mean absorption coefficient, we present calculations for the parameter $\mathrm{N}$ as a numerical measure of the relative importance of radiative transport to that of molecular conduction.

Figure 13 of Ref. 32 is reproduced here with the author's per-. mission as Fig. 17. In general, the absorption cross section varies extremely rapidly with frequency. Knowing the atomic structure of sodium, we see that the cross section above $1 \mu$ (the upper limit of experimental data) is effectively zero because of the lack of free-free, bound-free, or bound-bound transitions. For this reason, we agree with Wechsler ${ }^{33}$ that above $1 \mu$ the radiant energy absorbed by sodium vapor is negligible. Below $0.2 . \mu$, the thermal radiant energy absorbed is also negligible. This leaves the band between 0.2 and $1 \mu$, which is accounted for in the experimental work of Wechsler. ${ }^{33}$

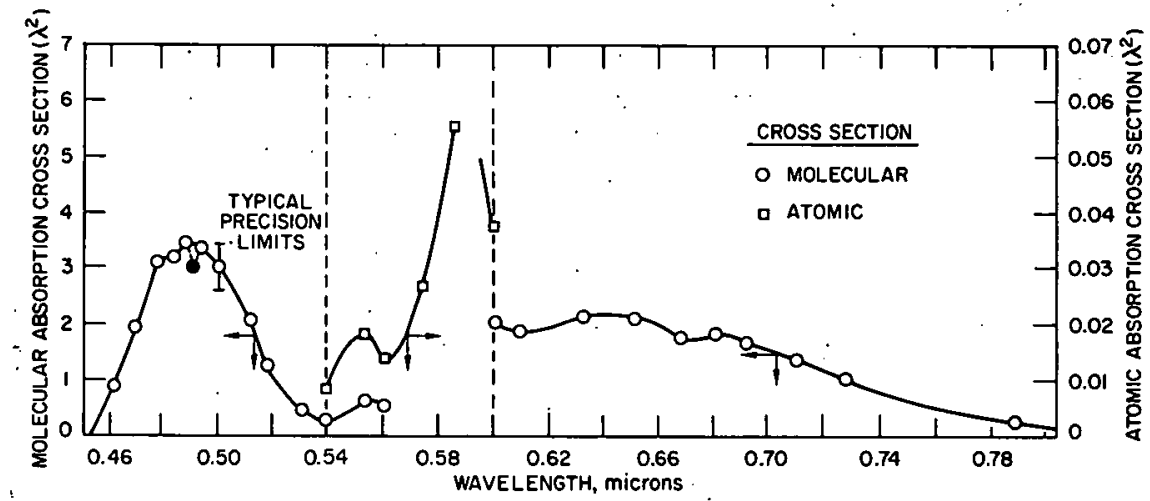

112-9203 Rev. 1

Fig. 17. Absorption Cross Section of Sodium Vapor ${ }^{32}$

The Planck-mean absorption coefficient is defined as ${ }^{1,2}$

$$
\kappa_{\mathrm{p}}=\frac{\int_{0}^{\infty} \mathrm{I}_{\mathrm{b}} \kappa_{\mathrm{r}} \mathrm{d} \nu}{\int_{0}^{\infty} \mathrm{I}_{\mathrm{b}} \mathrm{d} \nu}=\frac{\int_{0}^{\infty} \mathrm{I}_{\mathrm{b}} \kappa_{\mathrm{r}} \mathrm{d} \nu}{\frac{\sigma \mathrm{T}^{4}}{\pi}}
$$


where、

$$
\int_{0}^{\infty} I_{b} d \nu=\frac{\sigma T^{4}}{\pi}
$$

Before the integrations involved in Eq. A.l can be accomplished, data on the temperature dependence of sodium vapor must be available so that the absorption coefficient may be calculated from the absorption cross section. Values of the density as a function of temperature, as well as the vapor thermal conductivity, are taken from Ref. 34. The density varies by four orders of magnitude for a temperature change of approximately a factor of two: This wide variation of density with temperature appears to be a dominant factor in the analysis of the problem posed here.

Using the Romberg quadrature scheme; ${ }^{35,36}$ we integrate the numerator of Eq. A.1. The Romberg algorithm is essentially a trapezoidal integration of the function with a specified equal number of subintervals. From the first approximation, better approximations are calculated recursively. The ith iteration comprises the following: The ith value is calculated by summing the values over the mesh points of the ( $i-1)$ st iteration and additional points obtained by halving the previous mesh size. This sum is multiplied by the new mesh size. The $(i+1)$ st iteration is found by extrapolation from the ith and the previously calculated values. "Finally, the $(i+1)$ st value is compared with the ith value and the process is terminated if the absolute deviation is less than a preselected small value.

All computations are performed in double-precision arithmetic, and with the convergence criterion specified the integrals are at least as accurate as the data used, which are assumed valid to the second decimal place.

Representative values of the above computations are, for the boiling point of sodium at atmospheric pressure,

$$
\mathrm{T}=1619^{\circ} \mathrm{F}, \quad \kappa_{\mathrm{p}}=6.4 / \mathrm{cm}
$$

while at a higher temperature at atmospheric pressure,

$$
\mathrm{T}=2000^{\circ} \mathrm{F}, \quad \kappa_{\mathrm{p}}=40 \mathrm{l} / \mathrm{cm} \text {. }
$$

The mean absorption coefficient is an extremely nonlinear function of temperature. Thus, during transient boiling of a blanket of sodium, the medium may be optically thin initially because of the relatively "cool" temperature of the sodium and may become optically thick with time as the sodium absorbs energy and increases in temperature. The present analysis is primarily applicable for small time, however, and thus the approximations 
used here are valid in the sense that an optically thin medium would attenuate less radiant energy than an optically thick one and thus remain "cooler" relative to the walls, that is, emit a relatively smaller amount of radiant energy.

Using the above values of the Planck-mean absorption coefficient, computations for the parameter $N$ are performed using the definition

$$
N=\frac{k \kappa_{p}}{4 \sigma T_{i}^{4}},
$$

where the reference temperature $T_{i}$ is taken to be the evaluation temperature of the absorption coefficient: Thus at

$$
\mathrm{T}=1619^{\circ} \mathrm{F}, \quad \mathrm{N}=0.053 ;
$$

and at

$$
\mathrm{T}=2000^{\circ} \mathrm{F}, \quad \mathrm{N}=3.0 .
$$

Again, because of the strong temperature dependence of the Planck-mean absorption coefficient, $\kappa_{\mathrm{p}}$, the parameter $\mathrm{N}$ is strongly dependent on temperature. A more important conclusion, however, is the values of $\mathrm{N}$ indicate that radiation through sodium vapor is an important contributing mechanism to the total energy transport and should be accounted for. 


\section{APPENDIX B}

\section{Evaluation of Integrals.}

As was noted by Prasad, ${ }^{8}$ integrals of the form

$$
\int_{0}^{T_{1}} E_{n}(\tau) \cdot \sin \frac{n \pi \tau}{T_{0}} d \tau
$$

a.re inconvenient to evaluate explicitly; therefore he chose to use a different approximating function to solve the problem. The procedures for evaluating such integrals are outlined below. Integrating by parts, we obtain

$$
\begin{aligned}
& \int_{0}^{1} E_{4}\left(\tau_{0} \zeta\right) \sin (\mathrm{k} \pi \zeta) \mathrm{d} \zeta=\frac{-\mathrm{E}_{4}\left(\tau_{0}\right)(-1)^{\mathrm{k}}+\mathrm{E}_{4}(0)}{\mathrm{k} \pi} \\
& \quad-\left(\frac{\tau_{0}}{\mathrm{k} \pi}\right)^{2}\left[\frac{-\mathrm{E}_{2}\left(\tau_{0}\right)(-1)^{\mathrm{k}}+\mathrm{E}_{2}(0)}{\mathrm{k} \pi}\right]-\left(\frac{\tau_{0}}{\mathrm{k} \pi}\right)^{3}\left[\int_{0}^{1} \mathrm{E}_{1}\left(\tau_{0} \zeta\right) \cos (\mathrm{k} \pi \zeta) \mathrm{d} \zeta\right]
\end{aligned}
$$

Now;

$$
E_{1}\left(\tau_{0} \zeta\right)=-\Gamma-\ln \left(\tau_{0} \zeta\right)+\sum_{j=1}^{\infty}(-1)^{j-1} \frac{\left(\tau_{0} \zeta\right)^{j}}{j(j !)},
$$

where $\Gamma \equiv 0.577215$ (Euler's constant). Thus

$$
\begin{aligned}
& \int_{0}^{1} \mathrm{E}_{1}\left(\tau_{0} \zeta\right) \cos (\mathrm{k} \pi \zeta) \mathrm{d} \zeta=-\Gamma \int_{0}^{1} \cos (\mathrm{k} \pi \zeta) \mathrm{d} \zeta-\int_{0}^{1} \ln \left(\tau_{0} \zeta\right) \cos (\mathrm{k} \pi \zeta) \mathrm{d} \zeta \\
& \quad+\sum_{j=1}^{\infty} \frac{(-1) \mathrm{j}-1 \tau_{0} \mathrm{j}}{\mathrm{j}(\mathrm{j} !)} \int_{0}^{1} \zeta^{\mathrm{j}} \cos (\mathrm{k} \pi \zeta) \mathrm{d} \zeta
\end{aligned}
$$

The integrals in Eq. B.4, as given by Gradshteyn and Ryzhik, ${ }^{16}$ are 


$$
\begin{aligned}
& \int_{0}^{1} E_{1}\left(T_{0} \xi\right) \cos \mathrm{k} \pi \xi \mathrm{d} \xi=\frac{1}{\mathrm{k} \pi}\left[\operatorname{Si}\left(\frac{\mathrm{k} \pi}{T_{0}}\right)+\frac{\pi}{2}\right] \\
& +\sum_{j=1}^{\infty} \frac{(-1)^{j-1} \tau_{0}^{j}}{j(j !)}\left[\frac{(-1)^{k}}{k^{j+1}} \sum_{\ell=0}^{I^{\prime}\left(\frac{j-1}{2}\right)} \frac{(-1)^{\ell} j !}{(j-2 \ell-1) !}(k \pi)^{j-2 \ell-1}\right. \\
& \left.+(-1) I^{\prime} \frac{j+1}{2} \frac{2 I^{\prime}\left(\frac{j+l}{2}\right)-j}{(k)^{j+1}} \cdot j !\right]
\end{aligned}
$$

where the sine integral function has the series expansion:

$$
\operatorname{Si}(x) \equiv-\frac{\pi}{2}+\sum_{k=1}^{\infty} \frac{(-1)^{k+1} x^{2 k-1}}{(2 k-1)(2 k-1) !}
$$

and $I^{\prime}[(j-1) / 2] \equiv$ the integral part of the real number $x$. Finally, for the evaluation of

$$
\int_{0}^{1} \dot{E}_{4}\left[\tau_{0}(1-\xi)\right] \sin (\mathrm{k} \pi \xi) \mathrm{d} \xi
$$

let

$$
\mathrm{Z} \equiv 1-\xi
$$

and obtain

$$
-\int_{1}^{0} E_{4}\left(\tau_{0} \xi\right) \sin [k \pi(1-Z)] d Z
$$

which, after some trigonometric reduction, becomes

$$
=(-1)^{k-1} \cdot \int_{0}^{1} E_{4}\left(\tau_{0} Z\right) \sin (k \pi Z) d Z \text {, }
$$

which is tabulated as Eqs. B.2 and B.5. 


\section{ACKNOW LEDGMENTS}

I (Roger Heinisch) would like to thank Dr. Paul A. Lottes for his encouragement and support. I also wish to thank the Associated Midwest Universities for their support in the form of a fellowship. The assistance of the entire library staff, and, in particular, Mrs. Marion Benson, is gratefully acknowledged.

I also express my sincere thanks to Norman Jesse of the Applied Mathematics Division for assistance in running a check program on all calculations obtained. 
1. Cess, R. Do, The Interaction of Thermal Radiation with Conduction and Convection Heat Transfer, Advances in Heat Transfer, Vol. 1, Academic Press, New York (1964)。

2. Viskanta, R., Radiation Transfer and Interaction of Convection with Radiation Heat Transfer, Advances in Heat Transfer, Vol.: 3, New York: (1966).

3. Van der Held, E. F. M., The Contribution of Radiation to the Conduction of Heat, App1. Sci. Res., Sec。A, Vol。:3 (1951-1952).

4. Gardon, R., Calculation of Temperature Distributions in Glass Plates Undergoing Heat Treatment, J. Am. Ceram. Soc., Volo. 41, No. 6 (1958).

5. Zeldovich, Y. B., and Raizer, Y. P., Physics of Shock Waves and High Temperature Hydrodynamic Phenomena, Vol. 1, Academic Press (1966).

6. Lick, W., Transient Energy Transfer by Radiation and Conduction; Int. J. Heat and Mass Transfer, Vol. 8 (Feb 1965).

7. Nemchinov, I. V., Some Non-Stationary Problems of Radiative Heat Transfer, PMTF, No.], 1960 (in Russian) translated as Purdue University $A$ and ES TT -4 .

8. Prasad, K. K. Unsteady Radiant Heat Transfer in Stationary Media, $\mathrm{Ph} . \mathrm{D}$. thesis; University of Illinois (1966).

9. Viskanta, R:, and Bathla, P. S., Unsteady Energy Transfer in a Layer of Gray Gas by Thermal Radiation; J. App1. Math. and Phys. (ZAMP),

Vol. $18 \cdot(1967)$.

10. Cobble, M. H. : Irradiation into Transparent Solids and the Thermal Trap Effect, J. Franklin Inst。; Vol。,278, No. 6 (Dec,1964).

11. Viskanta, R., and Grosh, R. J., Heat Transfer by SimuZtaneous Conduction and Radiation in an Absorbing Medium, Trans. ASME J. Heat Transfer, Vo1. 84, Series C (1962).

12. Singer, R. M., Transient Two-phase Flow Following Sudden Vaporization, presented at the Symposium on the Dynamics of Two-phase Flow at Eindhoven; The Netherlands. (Sept. 4-9, 1967).

13. Holtz, R. E., and Singer, R. M., on the Superheating of Sodium and the Generation of Pressure Pulses; presented at International. Conference on the Safety of Fast Reactors, Aix-en-Provence, France (Sept. 19-22; 1967).

14. Sparrow, E. M., and Cess, R. D., Radiation Heat Transfer, Wadsworth. Publishing Co., Belmont, California (1966).

15. Kourganoff; V., Basic Methods of Transfer Problems, Dover Pub1. Inc. New York: (1963). 
16. Gradshteyn̈, I. S., and Ryzhi"k; I. Mo, Table of Integrals, Series, and Products, Academic Press, New York (1965).

17. Viskanta, R., Heat Transfer in Thermal Radiation Absorbing and Scattering Media; ANL-6170 (May 1960).

18. Omarov, E. 0., Approximate Solution by the Straight Line Method of a Partial Differential Equation of the First Elliptic Type, zh. vyh. mat., Vol. 4, No. 3 (1964) (in Russian).

19. Tien, C. L., Strip Method for Steady Heat Conduction ProbZems, App1. Sci. Res., Sect. A, Vo1. 13 (1964-1965).

20. Eyres, N. R., et al., The Calculation of Variable Heat Flow in Solids, Phil. Trans: Roy. Soc., London, A 240 (1946).

21. Dorodnitsyn, Solution of Mathematical and Logical Problems on High Speed Digital Computers, Sbornik, Theoretical Work on Aerodynamics, oberongiz, Moscow (1949).

22. Faddeeva, V: N., The Straight Line Method as Applied to Some Boundary Value Problems, Trud. Matem, in-ta Ahad. Nauk USSR, 28 (1949).

23. Kostynkovich, E. Kh., The Convergence of the Straight Line Method when Applied in Different Schemes to the Solution of Some Boundary Value Problems, Dok1, Akad. Nauk: USSR, 118, No. 3 (1958).

24. Koob, S。 Ji, Purdụe Unịversity, private communication。

25. Liebmann, G., Solution of Transient Heat Flow and Heat Transfer ProbZems by-ReZaxation, Brit. J. Appl. Phys., Vol. 6, No. 4 (1955).

26. Faddeeva, V. N., Computational Methods of Linear Algebra, Dover Pub1. Inc. (1959).

27. Richtmeyer, R. D., Difference Methods for Initial Value Problems, Interscience Publishers, Inc., New York'(1957).

28. Carslaw, H. S., and Jaege, J. C., Conduction of Heat.in Solids, Oxford University (1959).

29. Witte, L. C., Heat Transfer From a Sphere to Liquid Sodium During Forced Convection, ANL-7296 (Jan 1967).

30. Andriankin, E: Io, Calculation of a Spherical Thermal Wave Accounting for Radiation, (1iteral translation), Zh Eksperim. i Teor. Fiz., Vol. $25 \cdot(1958)$.

31. Viskanta, Ri, Concerming the Definitions of the Mean Absorption Coefficient; Int. J. Heat Mass Transfer:?(1967).

32. Wechsler, A. E., Characteristics of Metal Vapor, ARL 66-0004 (1966). 
33. Wechsler, A. E., A. D. Little Co., Cambridge, Mass., personal. communication.

34. Golden, G: H., and Tokar, J. V., Thermophysical Properties of Sodium, ANL-7.323 (Aug' 1967).

35. Thacher; H. C:; Remark on Algorithm.60, Comm. ACM.7 (1964).

36. Bover, F. L., et al.; New Aspects in Numerical Quadrature, Proceedings of Symposia in Applied Mathematics, Vol. XV.

37. Sarmin; E. N.; and Chudov, L. A.; On the Stability of the Numerical Integration of Systems of Ordinary Differential Equations Arising in the Use of the Straight:Line Method; $\mathrm{Zh}$. Vych. Mat: 3, No. 6. (1963). 